\title{
Determination of the strong coupling constant using inclusive jet cross section data from multiple experiments
}

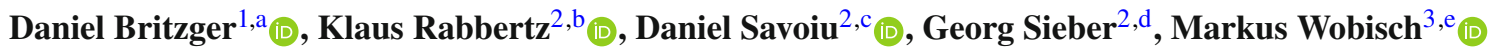 \\ ${ }^{1}$ Physikalisches Institut, Universität Heidelberg, Im Neuenheimer Feld 226, 69120 Heidelberg, Germany \\ ${ }^{2}$ Institut für Experimentelle Teilchenphysik (ETP), Karlsruhe Institute of Technology (KIT), Campus Süd, Postfach 6980, 76128 Karlsruhe, \\ Germany \\ ${ }^{3}$ Department of Physics, Louisiana Tech University, 600 Dan Reneau Dr., Ruston, LA, USA
}

Received: 24 July 2018 / Accepted: 21 December 2018 / Published online: 28 January 2019

(C) The Author(s) 2019

\begin{abstract}
Inclusive jet cross section measurements from the ATLAS, CDF, CMS, D0, H1, STAR, and ZEUS experiments are explored for determinations of the strong coupling constant $\alpha_{\mathrm{s}}\left(M_{\mathrm{Z}}\right)$. Various jet cross section data sets are reviewed, their consistency is examined, and the benefit of their simultaneous inclusion in the $\alpha_{\mathrm{s}}\left(M_{\mathrm{Z}}\right)$ determination is demonstrated. Different methods for the statistical analysis of these data are compared and one method is proposed for a coherent treatment of all data sets. While the presented studies are based on next-to-leading order in perturbative quantum chromodynamics (pQCD), they lay the groundwork for determinations of $\alpha_{\mathrm{s}}\left(M_{\mathrm{Z}}\right)$ at next-to-next-to-leading order.
\end{abstract}

\section{Introduction}

The strong coupling constant, $\alpha_{\mathrm{S}}$, is one of the least precisely known fundamental parameters in the Standard Model of particle physics. Because of its importance for precision phenomenology at the LHC and elsewhere, large efforts have been undertaken in the past decades to reduce uncertainties in determinations of $\alpha_{\mathrm{S}}$ [1-4].

With the advent of modern particle detectors and sophisticated algorithms for their simulation and calibration, jet measurements have become very precise. Many determinations of $\alpha_{\mathrm{s}}$ in deep-inelastic scattering (DIS) and in hadron-hadron collisions are therefore based on measurements of the inclusive jet cross section, which is directly proportional to $\alpha_{\mathrm{s}}$ in DIS in the Breit frame and $\alpha_{\mathrm{s}}^{2}$ in hadron-hadron collisions.

\footnotetext{
a e-mail: britzger@physi.uni-heidelberg.de

b e-mail: klaus.rabbertz@cern.ch

ce-mail: daniel.savoiu@cern.ch

de-mail: georg.sieber@cern.ch

e e-mail: wobisch@latech.edu
}

Using the most precise predictions of perturbative quantum chromodynamics (pQCD) available at the time, all previous $\alpha_{\mathrm{s}}$ extractions (except for Ref. [5]) were performed at next-to-leading order (NLO) in $\alpha_{\mathrm{s}}$. Their total uncertainty is dominated by the contribution related to the renormalisation scale dependence of the NLO pQCD results. The recent completion of next-to-next-to-leading order (NNLO) predictions for the inclusive jet cross section [6,7] promises a considerable reduction of the renormalisation scale dependence and will allow the inclusion of $\alpha_{\mathrm{S}}$ results from inclusive jet data in future determinations of the world average value of $\alpha_{\mathrm{s}}$ [1].

A determination of $\alpha_{\mathrm{s}}$ at NNLO from jet measurements in hadron-hadron collisions is still not readily achievable, because the new NNLO pQCD calculations are computationally very demanding and cannot yet be repeated quickly for different parton distribution functions (PDFs) or values of $\alpha_{\mathrm{S}}\left(M_{\mathrm{Z}}\right)$. In preparation of such a determination, it is desirable to study a simultaneous analysis of data sets from different processes and experiments. This study includes an investigation of the consistency of the various data sets and an estimation of the reduction of the experimental contributions to the $\alpha_{\mathrm{s}}$ uncertainty. The groundwork for these two aspects is presented in this article.

We review inclusive jet cross section data over a wide kinematic range, from different experiments for various initial states and centre-of-mass energies, and study their potential for determinations of $\alpha_{\mathrm{s}}$. The consistency of the diverse data sets is examined and the benefit of their simultaneous inclusion is demonstrated. Different methods for the statistical analysis of the data are compared and one method is proposed for a coherent treatment of all data sets in an extraction of $\alpha_{\mathrm{s}}\left(M_{\mathrm{Z}}\right)$.

The article is structured as follows: The experimental data sets and the theoretical predictions are introduced in Sects. 2 and 3, respectively. Methods and results from previous $\alpha_{\mathrm{s}}$ 
determinations by different experimental collaborations are discussed and employed in Sect. 4. The strategy for a determination of $\alpha_{\mathrm{s}}$ from multiple data sets and the final result are presented in Sect. 5.

\section{Experimental data}

The first measurement of the inclusive jet cross section has been performed in 1982 by the UA2 Collaboration at the Spp̄S collider at a centre-of-mass energy of $540 \mathrm{GeV}$ [8]. Further measurements have been conducted at centre-of-mass energies of

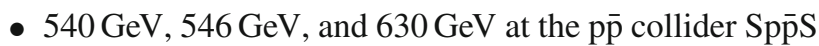
by the UA1 $[9,10]$ and UA2 experiments [11],

- $546 \mathrm{GeV}, 630 \mathrm{GeV}, 1.8 \mathrm{TeV}$, and $1.96 \mathrm{TeV}$ at the Tevatron $\mathrm{p} \overline{\mathrm{p}}$ collider by the CDF [12-17] and D0 experiments [1820],

- $300 \mathrm{GeV}$ and $320 \mathrm{GeV}$ at the ep collider HERA by the H1 [21-28] and ZEUS experiments [29-35],

- $200 \mathrm{GeV}$ in pp collisions at RHIC by the STAR experiment [36],

- and of $2.76 \mathrm{TeV}, 7 \mathrm{TeV}, 8 \mathrm{TeV}$, and $13 \mathrm{TeV}$ in pp collisions at the LHC by the ALICE [37], ATLAS [38-43], and CMS experiments [44-50].

While earlier measurements established the inclusive jet cross section as a useful quantity to study QCD, large experimental uncertainties limited their use for QCD phenomenology. When the NLO pQCD corrections were computed [5153], studies revealed collinear- or infrared-safety issues in the jet definitions used in the experimental measurements [54]. These issues were subsequently addressed and improved jet definitions were developed $[55,56]$ and applied in recent measurements.

Previously, $\alpha_{\mathrm{s}}\left(M_{\mathrm{Z}}\right)$ determinations were based on inclusive jet cross section data from individual experiments, as summarised in Table 1. An extraction of $\alpha_{\mathrm{S}}\left(M_{\mathrm{Z}}\right)$ from multiple inclusive jet cross section data sets has not been performed so far, except in the context of global PDF analyses, in which PDFs and $\alpha_{\mathrm{S}}\left(M_{\mathrm{Z}}\right)$ are determined simultaneously. These analyses, however, require data for a variety of measured quantities [21,57-60]. In this article, $\alpha_{\mathrm{s}}\left(M_{\mathrm{Z}}\right)$ is determined in a fit to multiple inclusive jet cross section measurements from experiments at HERA, RHIC, the Tevatron, and the LHC. The analysis is based on one selected measurement from each, the H1, ZEUS, STAR, CDF, D0, ATLAS, and CMS collaborations, as listed in Table 2.

Whenever experiments provide multiple measurements, we include those measured with a collinear- and infraredsafe jet algorithm $\left(k_{\mathrm{T}}[68]\right.$ or anti- $\left.k_{\mathrm{T}}[69]\right)$ and with a larger jet size parameter $R$, which improves the stability of fixed- order pQCD calculations. The STAR experiment published inclusive jet data collected by two different triggers with partially overlapping jet $p_{\mathrm{T}}$ ranges. We choose the data set collected with the trigger covering the higher jet $p_{\mathrm{T}}$ range from $7.6 \mathrm{GeV}$ up to $50 \mathrm{GeV}$. The measurements from the STAR and D0 experiments are using the midpoint cone jet algorithms (MP) [55]. The infrared-unsafety of this jet algorithm [54] prohibits NNLO pQCD predictions for these data sets, but it does not affect calculations at NLO. Four new measurements $[28,42,43,50]$ could not be included in this study; they are left for a future extension.

\section{Theoretical predictions and tools}

Predictions for the inclusive jet cross section in processes with initial-state hadrons are calculated as the convolution of the partonic cross section $\hat{\sigma}$ (computed in pQCD) and the PDFs of the hadron(s). The inclusive jet cross section in hadron-hadron collisions can be written as $[1,70]$

$$
\begin{aligned}
& \sigma_{\mathrm{PQCD}, \mathrm{hh}}\left(\mu_{\mathrm{r}}, \mu_{\mathrm{f}}\right)=\sum_{i, j} \int d x_{1} \int d x_{2} \\
& f_{i / h_{1}}\left(x_{1}, \mu_{\mathrm{f}}\right) f_{j / h_{2}}\left(x_{2}, \mu_{\mathrm{f}}\right) \hat{\sigma}_{i j \rightarrow \mathrm{jet}+X}\left(\mu_{\mathrm{r}}, \mu_{\mathrm{f}}\right),
\end{aligned}
$$

where the sum is over all combinations of parton flavors $i$ and $j$ (quarks, anti-quarks, and the gluon). The $f_{i, j / h_{1,2}}$ denote the PDFs for the parton flavours $i$ or $j$ in the initial-state hadrons $h_{1}$ and $h_{2}$, and $x_{1}$ and $x_{2}$ correspond to the fractional hadron momenta carried by the partons $i$ and $j$, respectively. The partonic cross section $\hat{\sigma}_{i j \rightarrow \text { jet }+X}$ is computed as a perturbative expansion in $\alpha_{\mathrm{s}}$ as

$\hat{\sigma}_{i j \rightarrow \mathrm{jet}+X}\left(\mu_{\mathrm{r}}, \mu_{\mathrm{f}}\right)=\sum_{n} \alpha_{s}^{n}\left(\mu_{\mathrm{r}}\right) c_{i j \rightarrow \mathrm{jet}+X}^{(n)}\left(\mu_{\mathrm{r}}, \mu_{\mathrm{f}}\right)$,

where the $c_{i j \rightarrow \mathrm{jet}+X}^{(n)}$ are computed from the pQCD matrix elements and the sum is over all orders of $\alpha_{\mathrm{s}}$ taken into account in the perturbative calculation. The renormalisation and factorisation scales are labelled $\mu_{\mathrm{r}}$ and $\mu_{\mathrm{f}}$, respectively. For inclusive jet production in hadron-hadron collisions, the first non-vanishing order (i.e. the leading order, LO) is given by $n=2$, while $n=3$ corresponds to the NLO corrections. For inclusive jet production in DIS in the Breit frame the partonic cross sections are convoluted with a single PDF and the LO (NLO) contribution is given by $n=1(n=2)$. Hence, inclusive jet production in $\mathrm{pp}, \mathrm{p} \overline{\mathrm{p}}$, and ep collisions is sensitive to $\alpha_{\mathrm{s}}$ already at LO .

For transverse jet momenta at the $\mathrm{TeV}$ scale accessible at the LHC, electroweak (EW) tree-level effects of $\mathcal{O}\left(\alpha \alpha_{\mathrm{s}}, \alpha^{2}\right)$ and loop effects of $\mathcal{O}\left(\alpha \alpha_{\mathrm{s}}^{2}\right)$ become sizeable [71]. A recent study of the complete set of QCD and EW NLO corrections has been presented in Ref. [72]. 
Table 1 Summary of previous determinations of $\alpha_{\mathrm{S}}\left(M_{\mathrm{Z}}\right)$ from inclusive jet cross sections. Rows 1-4 list the recent $\alpha_{\mathrm{s}}\left(M_{\mathrm{Z}}\right)$ extractions from double-differential inclusive jet cross sections by experimental collaborations that are studied in more detail in this work. Rows 5-12 and 13-16 summarise further determinations of $\alpha_{\mathrm{s}}\left(M_{\mathrm{Z}}\right)$ by experimental collaborations and by independent authors, respectively. The results in Refs. [61,67] are reported for approximate NNLO (aNNLO) and NLO used for the pQCD predictions. In Ref. [61] only 22 out of the 110 D0 data points were used in the $\alpha_{\mathrm{S}}\left(M_{\mathrm{Z}}\right)$ extraction; the decomposition of the uncertainties is only provided for the aNNLO result. In case of Ref. [66], we only consider scale, PDF, and NP related uncertainties as theoretical uncertainty for reasons of comparability to the other listed results

\begin{tabular}{|c|c|c|c|}
\hline Publication & Data & Comment & $\alpha_{\mathrm{s}}\left(M_{\mathrm{Z}}\right)$ \\
\hline H1 [27] & H1 [27] & HERA II, high $Q^{2}$ & $0.1174(22)_{\exp }(50)_{\text {theo }}$ \\
\hline D0 [61] & D0 [20] & aNNLO, 22 points & $0.1161\left({ }_{-33}^{+34}\right)_{\exp }\left({ }_{-35}^{+29}\right)_{\text {theo }}$ \\
\hline D0 [61] & D0 [20] & NLO, 22 points & $0.1202\left({ }_{-59}^{+72}\right)_{\text {tot }}$ \\
\hline CMS [62] & CMS [46] & $7 \mathrm{TeV}, 5.0 \mathrm{fb}^{-1}$ & $0.1185(19)_{\exp }\left({ }_{-37}^{+60}\right)_{\text {theo }}$ \\
\hline H1 [21] & H1 [21] & HERA I, $\sqrt{s}=300 \mathrm{GeV}$ & $0.1186(30)_{\exp }(51)_{\text {theo }}$ \\
\hline H1 [24] & H1 [24] & HERA I, $\sqrt{s}=320 \mathrm{GeV}$ & $0.1193(14)_{\exp }\left({ }_{-34}^{+50}\right)_{\text {theo }}$ \\
\hline H1 [26] & H1 [26] & HERA I, $\sqrt{s}=320 \mathrm{GeV}$, low $Q^{2}$ & $0.1180(18)_{\exp }\left({ }_{-93}^{+124}\right)$ theo \\
\hline H1 [5] & H1 [21,24,26-28] & HERA I+II, NNLO & $0.1152(20)_{\exp }(27)_{\text {theo }}$ \\
\hline ZEUS [30] & ZEUS [30] & $\sqrt{s}=300 \mathrm{GeV}, Q^{2}>500 \mathrm{GeV}^{2}$ & $0.1212\left({ }_{-35}^{+29}\right)_{\exp }\left({ }_{-27}^{+28}\right)$ theo \\
\hline ZEUS [33] & ZEUS [32] & $d \sigma / d Q^{2}, Q^{2}>500 \mathrm{GeV}^{2}$ & $0.1207\left({ }_{-36}^{+38}\right)_{\exp }\left({ }_{-23}^{+22}\right)_{\text {theo }}$ \\
\hline $\mathrm{CDF}[63]$ & $\mathrm{CDF}[63]$ & $1.8 \mathrm{TeV}, 87 \mathrm{pb}^{-1}$ & $0.1178\left({ }_{-95}^{+81}\right)_{\exp }\left({ }_{-75}^{+92}\right)$ theo \\
\hline CMS [50] & CMS [50] & $8 \mathrm{TeV}, 19.7 \mathrm{fb}^{-1}$ & $0.1164\left({ }_{-15}^{+14}\right)_{\exp }\left({ }_{-40}^{+59}\right)_{\text {theo }}$ \\
\hline Giele et al. [64] & CDF [65] & $1.8 \mathrm{TeV}, 4.2 \mathrm{pb}^{-1}$ & $0.121(8)_{\exp }(5)_{\text {theo }}$ \\
\hline Malaescu et al. [66] & ATLAS [39] & $7 \mathrm{TeV}, 37 \mathrm{pb}^{-1}$ & $0.1151(47)_{\exp }\left({ }_{-40}^{+51}\right)_{\text {theo }}$ \\
\hline Biekötter et al. [67] & H1 [27] & aNNLO & $0.122(2)_{\exp }(13)_{\text {theo }}$ \\
\hline Biekötter et al. [67] & H1 [27] & NLO & $0.115(2)_{\exp }(5)_{\text {theo }}$ \\
\hline
\end{tabular}

Table 2 Overview of the inclusive jet data sets used in the $\alpha_{\mathrm{s}}$ determinations. For each data set the process (proc), the centre-of-mass energy $\sqrt{s}$, the integrated luminosity $\mathcal{L}$, the number of data points, and the jet algorithm are listed. In case of ep collider data, the kinematic range may be defined by the four-momentum transfer squared $Q^{2}$, the inelasticity $y_{\text {DIS }}$, or the angle of the hadronic final state $\left|\cos \gamma_{h}\right|$ of the NC DIS process. In all cases, jets are required to be within a given range of pseudorapidity $\eta$ or rapidity $y$ in the laboratory frame

\begin{tabular}{|c|c|c|c|c|c|c|c|}
\hline Data & proc & $\sqrt{s}(\mathrm{TeV})$ & $\mathcal{L}\left(f b^{-1}\right)$ & No. of points & Jet algorithm & $p_{\mathrm{T}}, E_{\mathrm{T}}$-range $(\mathrm{GeV})$ & Other kinematic ranges \\
\hline H1 [27] & ep & 0.32 & 0.35 & 24 & $k_{\mathrm{T}}, R=1.0$ & $7<p_{\mathrm{T}}<50$ & $\begin{array}{c}150<Q^{2}<15000 \mathrm{GeV}^{2} \\
0.2<y_{\text {DIS }}<0.7 \\
-1.0<\eta<2.5\end{array}$ \\
\hline ZEUS [32] & ep & 0.32 & 0.082 & 30 & $k_{\mathrm{T}}, R=1.0$ & $E_{\mathrm{T}}>8$ & $\begin{array}{l}Q^{2}>125 \mathrm{GeV}^{2} \\
\left|\cos \gamma_{h}\right|<0.65 \\
-2.0<\eta<1.5\end{array}$ \\
\hline STAR [36] & $\mathrm{pp}$ & 0.20 & 0.0003 & 9 & $\mathrm{MP}, R=0.4$ & $7.6<p_{\mathrm{T}}<48.7$ & $0.2<|\eta|<0.8$ \\
\hline CDF [16] & $\mathrm{p} \overline{\mathrm{p}}$ & 1.96 & 1.0 & 76 & $k_{\mathrm{T}}, R=0.7$ & $54<p_{\mathrm{T}}<527$ & $|y|<2.1$ \\
\hline D0 [20] & $\mathrm{p} \overline{\mathrm{p}}$ & 1.96 & 0.7 & 110 & MP, $R=0.7$ & $50<p_{\mathrm{T}}<665$ & $|y|<2.0$ \\
\hline ATLAS [41] & $\mathrm{pp}$ & 7.0 & 4.5 & 140 & anti- $k_{\mathrm{T}}, R=0.6$ & $100<p_{\mathrm{T}}<1992$ & $|y|<3.0$ \\
\hline CMS [62] & $\mathrm{pp}$ & 7.0 & 5.0 & 133 & anti- $k_{\mathrm{T}}, R=0.7$ & $114<p_{\mathrm{T}}<2116$ & $|y|<3.0$ \\
\hline
\end{tabular}

Non-perturbative (NP) corrections to the cross section due to multiparton interactions and hadronisation can be estimated by using Monte Carlo (MC) event generators. An overview of MC event generators for the $\mathrm{LHC}$ is presented in Ref. [73]. The size of this correction depends on the jet size $R$, shrinks with increasing jet $p_{\mathrm{T}}$, and becomes negligible at the $\mathrm{TeV}$ scale. The total theory prediction for the inclusive jet cross section is given by $\sigma_{\text {theory }}=\sigma_{\mathrm{pQCD}} \cdot c_{\mathrm{EW}} \cdot c_{\mathrm{NP}}$,

where $c_{\mathrm{EW}}$ and $c_{\mathrm{NP}}$ are the correction factors for electroweak and non-perturbative corrections, respectively.

The partonic cross section is computed at NLO accuracy for five massless quark flavours using the NLOJet++ program version 4.1.3 $[74,75]$ within the fastNLO framework at version $2[76,77]$ to allow us fast recalculations for vary- 
ing PDFs, scales $\mu_{\mathrm{r}}$ and $\mu_{\mathrm{f}}$, and assumptions on $\alpha_{\mathrm{s}}\left(M_{\mathrm{Z}}\right)$. Jet algorithms are taken either from the FastJet software library [78] or, for jet cross sections in DIS, from NLOJet++. The PDFs are evaluated via the LHAPDF interface $[79,80]$ at version 6 . The running of $\alpha_{\mathrm{s}}\left(\mu_{\mathrm{r}}\right)$ is performed at 2-loop order using the package CRunDec with five massless quark flavours $[81,82]$. The minimal subtraction $(\overline{\mathrm{MS}})$ scheme [83-85] has been adopted for the renormalisation procedure in these calculations.

For the computation of the inclusive jet cross section in hadron-hadron collisions, the renormalisation and factorisation scales, $\mu_{\mathrm{r}}$ and $\mu_{\mathrm{f}}$, are identified with each jet's $p_{\mathrm{T}}$, i.e. $\mu_{\mathrm{r}}=\mu_{\mathrm{f}}=p_{\mathrm{T}}^{\text {jet }}$. In neutral current (NC) DIS, the scales are chosen to be $\mu_{\mathrm{r}}^{2}=\frac{1}{2}\left(Q^{2}+\left(p_{\mathrm{T}}^{\text {jet }}\right)^{2}\right)$ and $\mu_{\mathrm{f}}^{2}=Q^{2}$ as used by the H1 Collaboration [27]. Alternative scale choices have been discussed with respect to NNLO predictions $[5,7,86,87]$, but are beyond the scope of this article.

The EW corrections, $c_{\mathrm{EW}}$, relevant for the LHC data are provided by the experimental collaborations together with the data, based on Ref. [71]. These are considered to have negligible uncertainties. Due to restrictions of the scale choices in this calculation, the leading jet's transverse momentum, $p_{\mathrm{T}}^{\max }$, is used to define the scales $\mu_{\mathrm{r}}$ and $\mu_{\mathrm{f}}$. The NP correction factors $c_{\mathrm{NP}}$, except for the STAR data [88], are also provided by the experimental collaborations, together with an estimate of the corresponding uncertainty $[16,20,27,32,33,41,46,61,62]$.

\section{Comparison of three extraction methods for $\alpha_{\mathrm{s}}\left(M_{\mathrm{Z}}\right)$}

Commonly, the value of $\alpha_{\mathrm{S}}\left(M_{\mathrm{Z}}\right)$ is determined from inclusive jet cross sections in a comparison of $\mathrm{pQCD}$ predictions to the measurements. These $\alpha_{\mathrm{S}}\left(M_{\mathrm{Z}}\right)$ results therefore depend on details of the extraction method such as the treatment of uncertainties in the characterisation of differences between theory and data, or the evaluation and propagation of theoretical uncertainties to the final result. An overview of previous determinations of $\alpha_{\mathrm{S}}\left(M_{\mathrm{Z}}\right)$ from fits to inclusive jet cross section data is provided in Table 1 . We choose the three $\alpha_{\mathrm{s}}\left(M_{\mathrm{Z}}\right)$ determinations performed by the CMS [62], D0 [61], and H1 [27] collaborations listed in the upper part of Table 1 for further study.

The three extraction methods differ in the following aspects:

- the definition of the $\chi^{2}$ function to quantify the agreement between theory and data,

- the uncertainties considered in the $\chi^{2}$ function,

- the strategy to determine the central result for $\alpha_{\mathrm{s}}\left(M_{\mathrm{Z}}\right)$,

- the propagation of the uncertainties to the value of $\alpha_{\mathrm{s}}\left(M_{\mathrm{Z}}\right)$

- the choice of PDF sets,
- the consideration of the $\alpha_{\mathrm{S}}\left(M_{\mathrm{Z}}\right)$ dependence of the PDFs, and

- the treatment of further theoretical uncertainties.

To study the impact of these differences, we have implemented the three methods in our computational framework and will refer to them as "CMS-type", "D0-type", and "H1type", respectively. As an integral part of these methods and to reproduce as precisely as possible the respective published results, we restrict ourselves in this section to the use of the original somewhat older PDF sets. The CMS result was obtained with the CT10 PDF set [89], and the D0 and H1 results with MSTW2008 PDFs [90]. The CMS-type and D0type methods use the entire $\alpha_{\mathrm{s}}^{\mathrm{PDF}}\left(M_{\mathrm{Z}}\right)$ series available for the PDF set, whereas the H1-type method uses a PDF determined with a value of $\alpha_{\mathrm{s}}^{\mathrm{PDF}}\left(M_{\mathrm{Z}}\right)=0.1180$.

Each method is then employed to extract $\alpha_{\mathrm{S}}\left(M_{\mathrm{Z}}\right)$ from each of the individual data sets selected in Sect. 2, cf. also Table 2. The experimental uncertainties and their correlations are treated according to the respective prescriptions by the experiments. The resulting $\alpha_{\mathrm{S}}\left(M_{\mathrm{Z}}\right)$ values are listed in Table 3.

In a first step, these results are compared to the ones obtained by the CMS [62], D0 [61], and H1 [27] collaborations as listed in Table 1. All three central results are reproduced, the $\mathrm{H} 1$ result exactly, and the CMS and D0 results within +0.0003 and +0.0001 . Such small differences can easily be caused already by using different versions of LHAPDF (e.g. changes from version 5 to version 6). The experimental uncertainties of the CMS and $\mathrm{H} 1$ analyses are exactly reproduced. ${ }^{1}$

In a second step, the $\alpha_{\mathrm{S}}\left(M_{\mathrm{Z}}\right)$ results and their experimental uncertainties are compared to each other and their dependencies on the extraction method and PDFs are studied. The $\alpha_{\mathrm{S}}\left(M_{\mathrm{Z}}\right)$ results determined for each data set are displayed in Fig. 1 (top row) for the three different extraction methods using CT10 PDFs (left) and MSTW2008 PDFs (right). For the STAR data, $\alpha_{\mathrm{s}}\left(M_{\mathrm{Z}}\right)$ results cannot be determined in case of the CMS-type and D0-type methods with MSTW2008 PDFs, since no local $\chi^{2}$ minima are found. In all other cases the $\alpha_{\mathrm{S}}\left(M_{\mathrm{Z}}\right)$ results obtained with MSTW2008 PDFs are rather independent of the extraction method for all data sets. This is different when using CT10 PDFs: While in this case the extraction method has little impact on the $\alpha_{\mathrm{S}}\left(M_{\mathrm{Z}}\right)$ results from HERA data (H1 and ZEUS), it notably affects the results for the LHC data (ATLAS and CMS), and has large effects for the Tevatron data (CDF and D0). In the

\footnotetext{
${ }^{1}$ For the D0 analysis, the decomposition of uncertainties has been published only for their central result based on approximate NNLO pQCD and hence a comparison of the experimental uncertainty on $\alpha_{\mathrm{s}}\left(M_{\mathrm{Z}}\right)$ for the NLO result is not possible.
} 
Table 3 Values of $\alpha_{\mathrm{S}}\left(M_{\mathrm{Z}}\right)$ with experimental uncertainties obtained using the three extraction methods CMS-type, D0-type, and H1-type together with the CT10 or MSTW2008 PDF set at NLO . The under- lined values can be directly compared with the results published in Refs. $[27,61,62]$. Some fits to the STAR data do not exhibit a local minimum, in which case no value is listed

\begin{tabular}{|c|c|c|c|c|c|c|}
\hline \multirow{2}{*}{$\begin{array}{l}\text { Fit method } \\
\text { PDF set }\end{array}$} & \multicolumn{2}{|l|}{ CMS-type } & \multicolumn{2}{|l|}{ D0-type } & \multicolumn{2}{|l|}{ H1-type } \\
\hline & MSTW2008 & CT10 & MSTW2008 & CT10 & MSTW2008 & CT10 \\
\hline Data & \multicolumn{6}{|c|}{$\alpha_{\mathrm{s}}\left(M_{\mathrm{Z}}\right)$ values with experimental uncertainties } \\
\hline H1 & $0.1172(28)$ & $0.1172(28)$ & $0.1161(27)$ & $0.1164(26)$ & $0.1174(22)$ & $\underline{0.1180}(22)$ \\
\hline ZEUS & $0.1213(28)$ & $0.1223(29)$ & $0.1210\left({ }_{-29}^{+28}\right)$ & $0.1218\left({ }_{-29}^{+30}\right)$ & $0.1231(30)$ & $0.1236(30)$ \\
\hline STAR & - & $0.1193(68)$ & - & $0.1205\left({ }_{-111}^{+54}\right)$ & $0.1159(116)$ & $0.1280(111)$ \\
\hline $\mathrm{CDF}$ & $0.1217(17)$ & $0.1265(27)$ & $0.1202\left({ }_{-27}^{+10}\right)$ & $0.1162\left({ }_{-20}^{+22}\right)$ & $0.1217(35)$ & $0.1265(37)$ \\
\hline D0 (22 pts., NLO) & $0.1226(32)$ & $0.1237(36)$ & $\underline{0.1203}\left({ }_{-42}^{+40}\right)$ & $0.1191\left({ }_{-45}^{+38}\right)$ & $0.1219(50)$ & $0.1232(51)$ \\
\hline ATLAS & $0.1220(9)$ & $0.1258(15)$ & $0.1204\left({ }_{-5}^{+14}\right)$ & $0.1241(9)$ & $0.1206(15)$ & $0.1270(16)$ \\
\hline CMS & $0.1162(14)$ & 0.1188 (19) & $0.1158(12)$ & $0.1162(19)$ & $0.1140(21)$ & $0.1217(23)$ \\
\hline
\end{tabular}

latter cases, the D0-type method produces significantly lower $\alpha_{\mathrm{S}}\left(M_{\mathrm{Z}}\right)$ results as compared to the other two methods.

The $\chi^{2} / n_{\text {dof }}$ values for the $\alpha_{\mathrm{S}}\left(M_{\mathrm{Z}}\right)$ extractions are displayed in Fig. 1 (bottom row) for the three extraction methods using CT10 PDFs (left) and MSTW2008 PDFs (right). Overall, the fits exhibit reasonable values of $\chi^{2} / n_{\text {dof }}$, thus indicating agreement between theory and data. Exceptions are observed for the ZEUS data with rather low values of $\chi^{2} / n_{\text {dof }}$, and for the ATLAS data, where the values of $\chi^{2} / n_{\text {dof }}$ are large as also observed elsewhere [42,91,92].

The PDF dependence is displayed in Fig. 2, where the $\alpha_{\mathrm{S}}\left(M_{\mathrm{Z}}\right)$ results for CT10 and MSTW2008 PDFs are compared to each other, both obtained using the H1-type method. While the PDF choice has no significant effect for the results from the H1, ZEUS, and D0 data, smaller variations are seen for the CDF data, and a large dependence for the ATLAS and CMS data. Re-investigating this PDF dependence in the context of a common determination of $\alpha_{\mathrm{S}}\left(M_{\mathrm{Z}}\right)$ as described in the next section, we observe that differences with respect to the updated PDF sets, CT14 [93] and MMHT2014 [60], are reduced.

\section{Determination of $\alpha_{\mathrm{S}}\left(M_{\mathrm{Z}}\right)$ from multiple inclusive jet data sets}

The analysis of multiple data sets requires their correlations to be taken into account. For the present study, measurements from different colliders are considered to be uncorrelated because of the largely complementary kinematic ranges of the data sets and different detector calibration techniques. Furthermore, investigations with respect to $\mathrm{H} 1$ and ZEUS data [58], CDF and D0 data [94], or ATLAS and CMS data $[95,96]$, did not identify a relevant source of experimental correlation. This only leaves theoretical uncertainties as a source of potential correlations in this study. For the determination of NP effects and their uncertainties vari- ous methods and MC event generators have been employed $[16,20,27,32,36,41,61,62,88]$. While a consistent derivation of these corrections with corresponding correlations is desirable, this is beyond the scope of this analysis. Hence, the NP correction factors and their uncertainties are considered to be uncorrelated between the different data sets. In contrast, the PDF uncertainties and the uncertainties due to the renormalisation and factorisation scale variations are treated as fully correlated; the relative variations with respect to the nominal scales are performed simultaneously for all data sets.

The method employed for the simultaneous $\alpha_{\mathrm{s}}$ extraction combines components of the individual methods outlined in the previous section and is referred to as "CMStype method". The central $\alpha_{\mathrm{S}}\left(M_{\mathrm{Z}}\right)$ result is found in an iterative $\chi^{2}$ minimisation procedure adopted from the H1type method, where a normal distribution is assumed for the relative uncertainties. The exact $\chi^{2}$ formula is given by Eq. (4) of Appendix A. Whereas in the H1-type $\chi^{2}$ expression, only experimental uncertainties are taken into account, the common-type method also accounts for the NP and PDF uncertainties in the $\chi^{2}$ expression, as in the CMS-type and D0-type methods. This $\chi^{2}$ definition treats variances as relative values and thus has advantages, e.g. when numerically inverting the covariance matrix. Moreover, uncertainties of experimental and theoretical origin are put on an equal footing. As in the H1-type method, and in contrast to the CMStype and D0-type ones, only PDF sets obtained with a fixed value of $\alpha_{\mathrm{S}}\left(M_{\mathrm{Z}}\right)=0.1180$ are employed in the determination of the central $\alpha_{\mathrm{s}}\left(M_{\mathrm{Z}}\right)$ result, leaving the $\alpha_{\mathrm{s}}$ dependence of the PDFs to be treated as a separate uncertainty.

In contrast to Sect. 4, where the three investigated methods were introduced, we do not need to restrict ourselves to PDF sets available at the time of their publication. Instead we take advantage from significant progress in the PDF determination with respect to theoretical ingredients, computational techniques, and data input and consider the following 

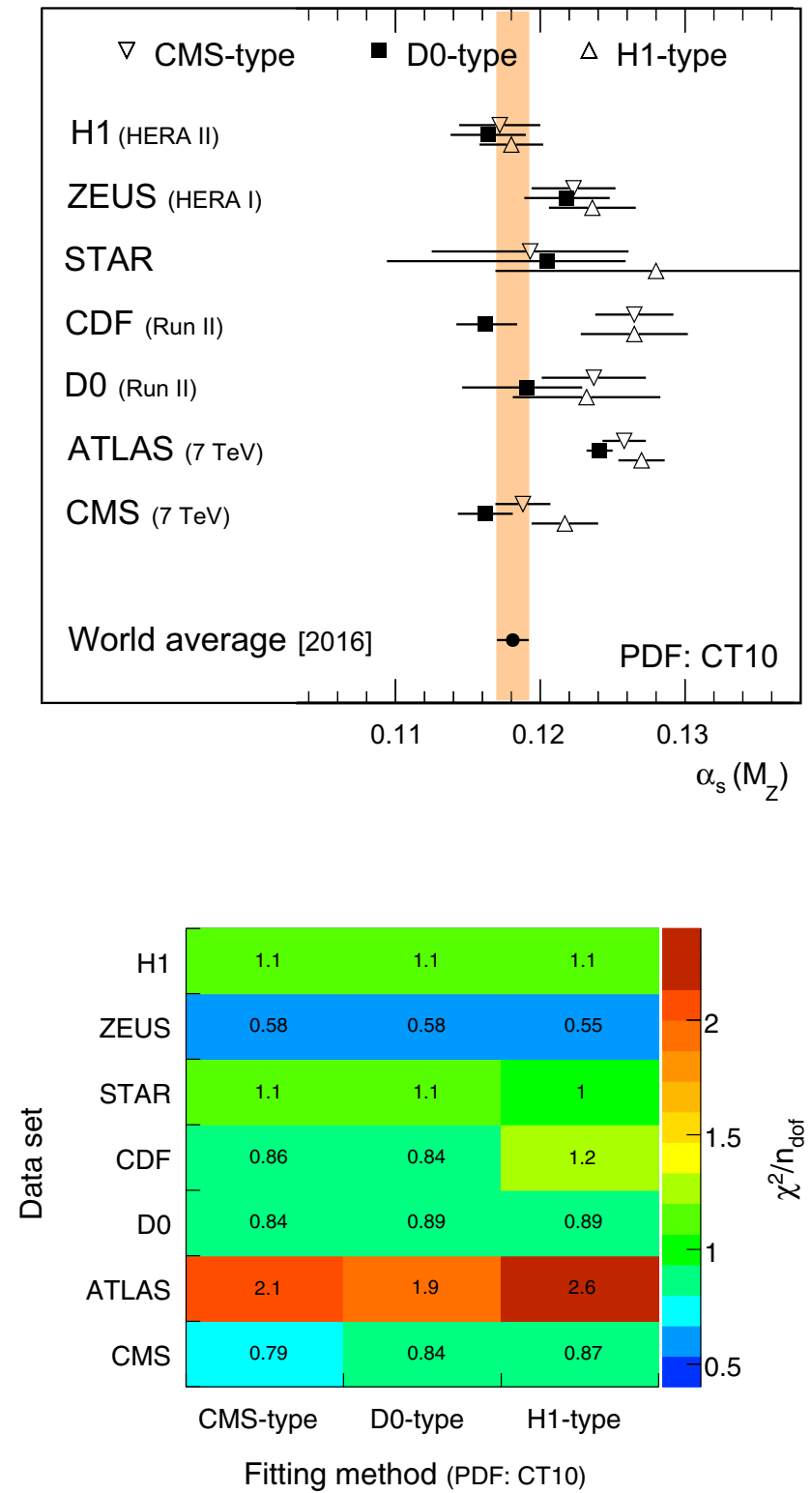

Fig. 1 Values of $\alpha_{\mathrm{S}}\left(M_{\mathrm{Z}}\right)$ with experimental uncertainties obtained using the three extraction methods CMS-type, D0-type, and H1-type. The upper row compares the results for each data set employing the PDF set CT10 (left) or MSTW2008 (right). In addition, the world average value [1] is shown together with a band representing its uncertainty. The

newer PDF sets ABMP16 [97,98], CT14 [93], HERAPDF2.0 [58], MMHT2014 [60], and NNPDF3.0 [99]. Since in case of CT14, MMHT2014, and NNPDF3.0 also jet data we analyse here have been used, the effect of potential correlations due to the dual usage of the data has been studied in Appendix B, where additional five variants of NNPDF3.0 [99] with different input data selections are employed. The effect of such correlations is found to be small and is covered by an additional uncertainty as defined below.

In summary, the individual contributions to the total uncertainty of the $\alpha_{\mathrm{s}}\left(M_{\mathrm{Z}}\right)$ result are evaluated as follows: The
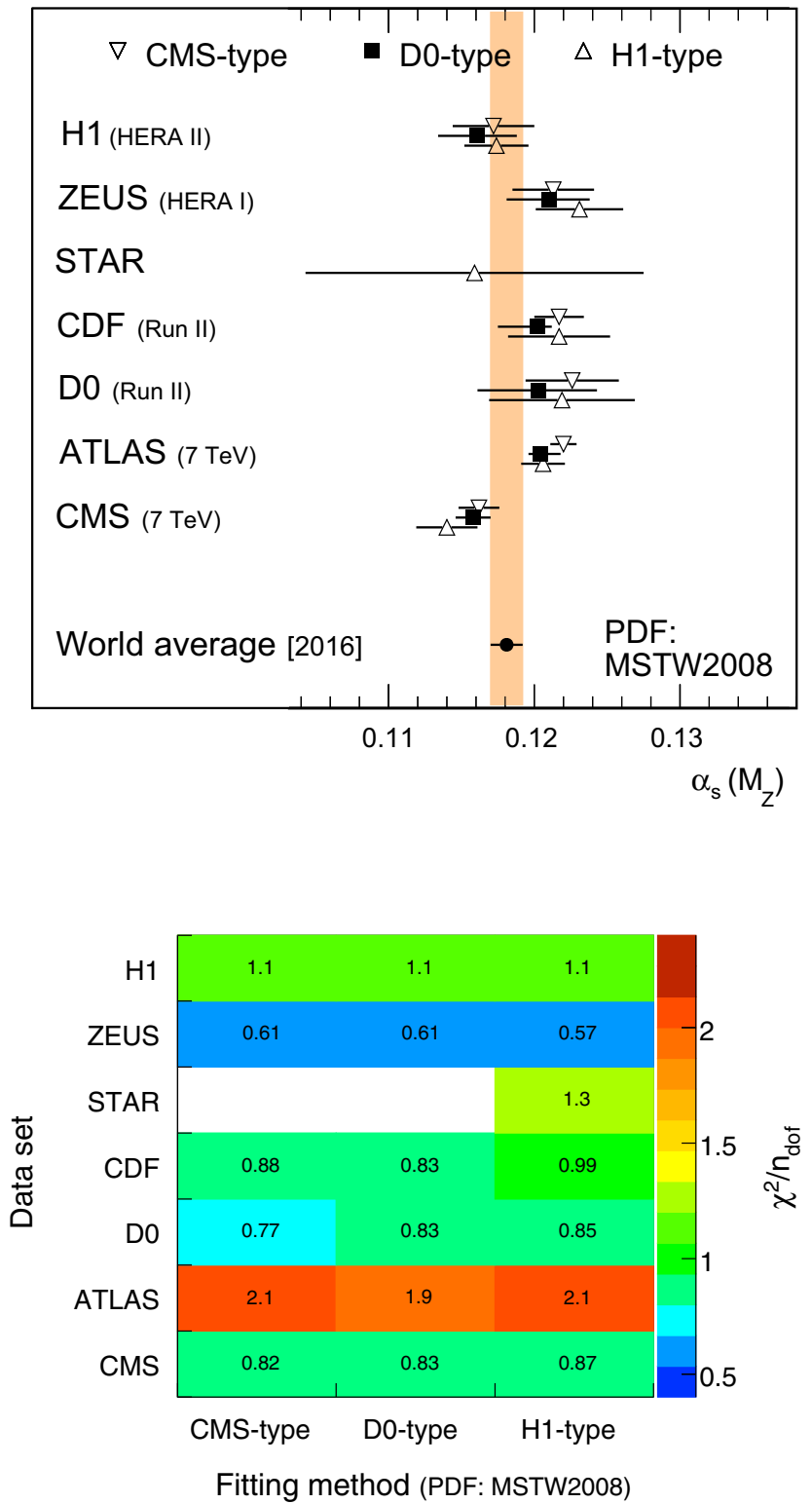

bottom row displays the values of $\chi^{2} / n_{\text {dof }}$ for each fit using the CT10 (left) or MSTW2008 PDF set (right). The colours illustrate the values of $\chi^{2} / n_{\text {dof }}$. For the STAR data and the MSTW2008 PDF set no local minimum was found in case of the CMS-type and D0-type fits (blank areas)

experimental uncertainty (exp) is obtained from the Hesse algorithm [100] when performing the $\alpha_{\mathrm{S}}\left(M_{\mathrm{Z}}\right)$ extraction with only the uncertainties of the measurements included. The NP and PDF uncertainties are derived by repeating the $\alpha_{\mathrm{S}}\left(M_{\mathrm{Z}}\right)$ extraction while successively including the corresponding uncertainty contributions and calculating the quadratic differences. Further sources of systematic effects are considered as follows:

- The "PDF $\alpha$ " "uncertainty accounts for the initial assumption of $\alpha_{\mathrm{s}}^{\mathrm{PDF}}\left(M_{\mathrm{Z}}\right)=0.1180$ made in the PDF extrac- 


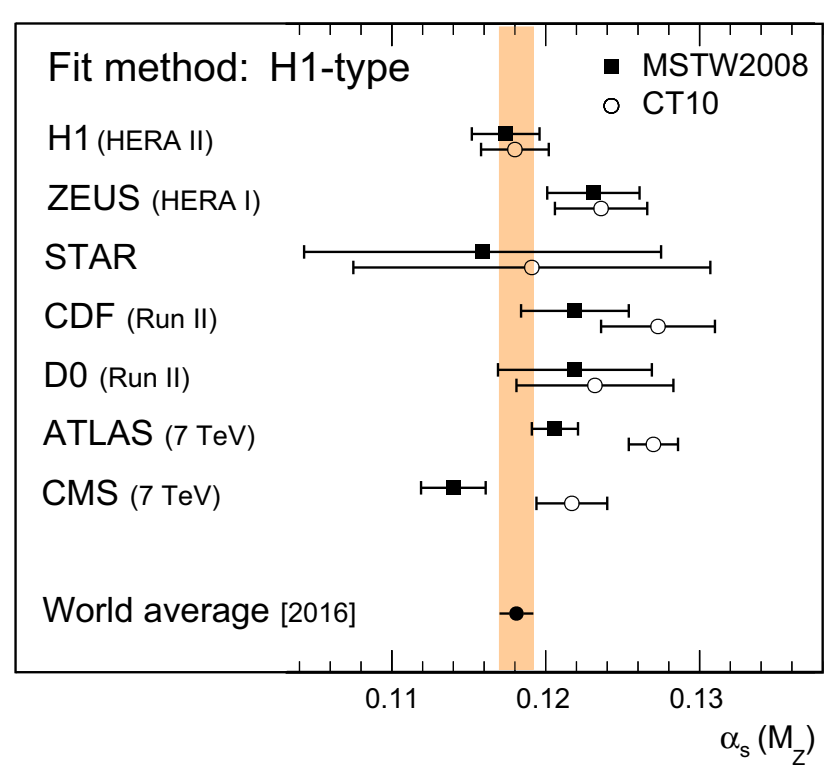

Fig. 2 Comparison of the $\alpha_{\mathrm{S}}\left(M_{\mathrm{Z}}\right)$ results with their experimental uncertainties obtained using the H1-type extraction methods for CT10 and MSTW2008 PDFs. The world average value [1] is shown together with a band representing its uncertainty

tion, which is not necessarily consistent with the value of $\alpha_{\mathrm{S}}\left(M_{\mathrm{Z}}\right)$ used in the pQCD calculation. It is calculated as the maximal difference between any of the results obtained with PDF sets determined for $\alpha_{\mathrm{s}}^{\mathrm{PDF}}\left(M_{\mathrm{Z}}\right)=$ $0.1170,0.1180$, and 0.1190 , and therefore covers a difference of $\Delta \alpha_{\mathrm{s}}^{\mathrm{PDF}}\left(M_{\mathrm{Z}}\right)=0.0020$, which is somewhat more conservative than the recommendation in Ref. [101].

- The "PDFset" uncertainty covers differences due to the considered PDF set. These are caused by the data selection, assumptions made on parameterisation, parameter

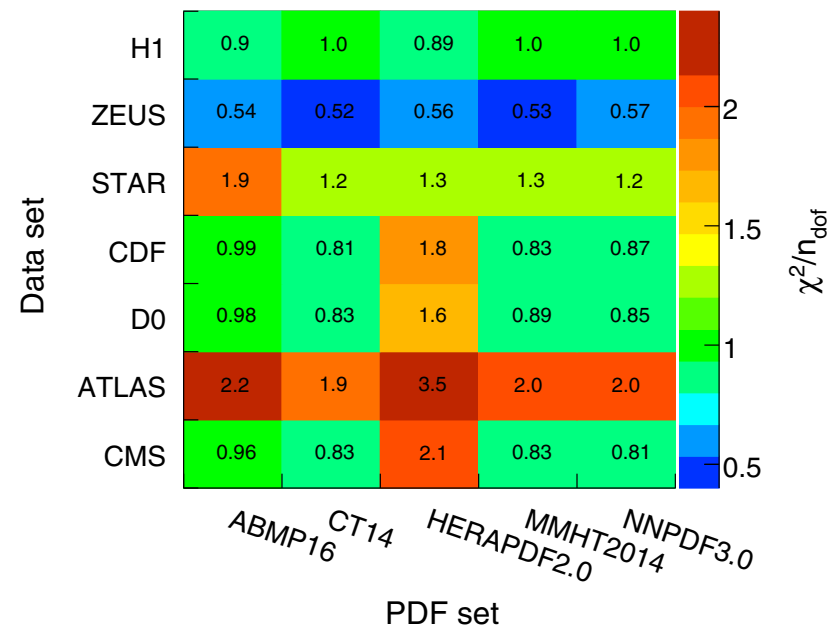

Fig. 3 Left: Illustration of the $\chi^{2} / n_{\text {dof }}$ values for fits to each data set individually. Right: Illustration of the $\chi^{2} / n_{\text {dof }}$ values for simultaneous fits omitting a single data set at a time. The included or respectively values, theory input, or the analysis method for the PDF determination. It also comprises the potential effect of the dual use of jet cross sections, first in the PDF determination and then in the fit of $\alpha_{\mathrm{S}}\left(M_{\mathrm{Z}}\right)$. Therefore, we define it as half of the width of the envelope constructed from the five values for the ABMP16, CT14, MMHT2014, and NNPDF3.0_nojet PDFs together with the NNPDF3.0 result. These global PDF sets either comprise a maximum of data other than jet cross sections or no jet cross sections at all.

- The uncertainty due to variations of the renormalisation and factorisation scales customarily is taken as an estimate for the error of a fixed-order calculation caused by the truncation of the perturbative series. It is obtained using six additional $\alpha_{\mathrm{S}}\left(M_{\mathrm{Z}}\right)$ determinations, in which the nominal scales $\left(\mu_{\mathrm{r}}, \mu_{\mathrm{f}}\right)$ are varied by the conventional factors of $(1 / 2,1 / 2),(1 / 2,1),(1,1 / 2),(1,2),(2,1)$, and $(2,2)$. The scale factor combinations of $(1 / 2,2)$ and $(2,1 / 2)$ are customarily omitted [102-104].

The NP, PDF, PDF $\alpha_{\mathrm{s}}$, PDFset, and scale uncertainties are added in quadrature to give the theoretical uncertainty (theo). The total uncertainty (tot) further includes the experimental uncertainty.

In the previous section, cf. Fig. 1, it was found that the $\chi^{2} / n_{\text {dof }}$ values differ significantly from unity for some of the data sets. This necessitates to investigate in further detail the consistency of the data within an individual data set as well as among the different data sets. Therefore, the commontype method is employed to extract $\alpha_{\mathrm{S}}\left(M_{\mathrm{Z}}\right)$ from each individual data set and for each of the PDFs ABMP16, CT14, HERAPDF2.0, MMHT2014, and NNPDF3.0. The resulting $\chi^{2} / n_{\text {dof }}$ values are displayed in Fig. 3 left. Detailed listings

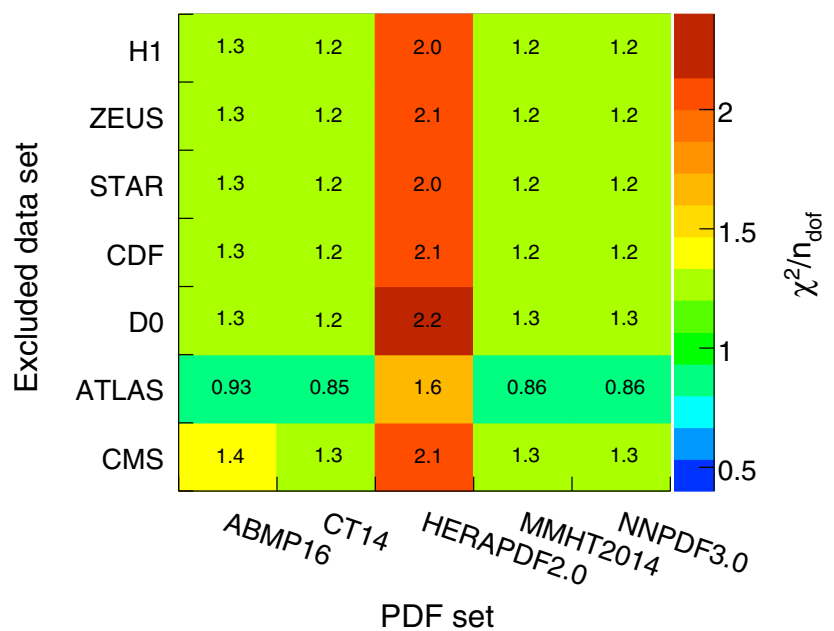

excluded data set is indicated on the $y$ axis and the PDF set on the $x$ axis. The fits are performed for each PDF set in the envelope definition of the PDFset uncertainty 
Table 4 Values of $\alpha_{\mathrm{s}}\left(M_{\mathrm{Z}}\right)$ for the simultaneous fit to the $\mathrm{H} 1$, ZEUS, STAR, CDF, D0, and CMS data using the common-type method for various PDF sets. The experimental, NP, PDFset, and scale uncertainties remain mostly unchanged under a change of the PDF set and are quoted only once for NNPDF3.0

\begin{tabular}{|c|c|c|c|c|c|c|c|c|c|}
\hline \multirow[t]{2}{*}{ PDF set } & \multirow[t]{2}{*}{$\alpha_{\mathrm{s}}\left(M_{\mathrm{Z}}\right)$} & \multicolumn{8}{|c|}{ Uncertainties (scaled by a factor of $10^{4}$ ) } \\
\hline & & $\exp$ & NP & PDF & $\mathrm{PDF} \alpha_{\mathrm{s}}$ & PDFset & scale & theo & total \\
\hline ABMP16 & 0.1203 & & & 4 & 3 & & & $\begin{array}{l}+63 \\
-46\end{array}$ & $\begin{array}{l}+64 \\
-48\end{array}$ \\
\hline CT14 & 0.1206 & & & 10 & 2 & & & $\begin{array}{l}+57 \\
-46\end{array}$ & $\begin{array}{l}+59 \\
-48\end{array}$ \\
\hline HERAPDF2.0 & 0.1184 & & & 6 & 2 & & & $\begin{array}{l}+62 \\
-50\end{array}$ & $\begin{array}{l}+63 \\
-52\end{array}$ \\
\hline ММHТ2014 & 0.1194 & & & 7 & 3 & & & $\begin{array}{r}+59 \\
-45\end{array}$ & $\begin{array}{l}+60 \\
-47\end{array}$ \\
\hline NNPDF3.0_nojet & 0.1196 & & & 12 & 9 & & & $\begin{array}{l}+60 \\
-44\end{array}$ & $\begin{array}{l}+61 \\
-46\end{array}$ \\
\hline NNPDF3.0 & 0.1192 & 12 & 5 & 7 & 5 & 7 & $\begin{array}{l}+59 \\
-38\end{array}$ & $\begin{array}{l}+60 \\
-40\end{array}$ & $\begin{array}{l}+61 \\
-42\end{array}$ \\
\hline
\end{tabular}

of the $\alpha_{\mathrm{S}}\left(M_{\mathrm{Z}}\right)$ results and their uncertainties are given in Appendix B.

For a given data set, $\chi^{2} / n_{\text {dof }}$ is rather independent of the PDF set used for the predictions and varies between 0.8 and 1.2. These values indicate reasonable agreement of the predictions with the data. Exceptions are rather low values of $\chi^{2} / n_{\text {dof }}$ around 0.54 found for all PDF sets with the ZEUS data and large $\chi^{2} / n_{\text {dof }}$ values between 1.9 and 3.5 exhibited by the ATLAS data, also for all PDFs. Exceptionally large $\chi^{2} / n_{\text {dof }}$ values appear for the Tevatron or LHC data together with theory predictions using the HERAPDF2.0 set, and for the STAR data in conjunction with the ABMP16 PDF set.

To further investigate the consistency among the data sets, a series of $\alpha_{\mathrm{S}}\left(M_{\mathrm{Z}}\right)$ extractions is performed, in which $\alpha_{\mathrm{S}}\left(M_{\mathrm{Z}}\right)$ is determined simultaneously from all data sets but one. This is repeated for each PDF set. The resulting $\chi^{2} / n_{\text {dof }}$ values are displayed in Fig. 3 right. Apparently, the exclusion of the ATLAS data leads to significantly smaller $\chi^{2} / n_{\text {dof }}$ values independent of the PDF set used. This hints at a compatibility issue when using all data sets together, which is not present when the ATLAS data set is ignored. Therefore, we choose to exclude the ATLAS data for our main result, which is thus obtained from the CDF, CMS, D0, H1, STAR, and ZEUS inclusive jet data. The choice of the NNPDF3.0 set for the central result yields

$$
\begin{aligned}
\alpha_{\mathrm{s}}\left(M_{\mathrm{Z}}\right)= & 0.1192(12)_{\exp }(5)_{\mathrm{NP}} \\
& (7)_{\mathrm{PDF}}(5)_{\mathrm{PDF}} \alpha_{\mathrm{s}}(7)_{\mathrm{PDFset}}\left({ }_{-38}^{+59}\right)_{\text {scale }},
\end{aligned}
$$

with $\chi^{2}=328$ for 381 data points. This result is consistent with the world average value of 0.1181 (11) [1], which has been derived exclusively using QCD theory at NNLO or higher. The experimental uncertainty for the extraction from multiple data sets is significantly smaller than each of the experimental uncertainties reported previously for the separate $\alpha_{\mathrm{s}}\left(M_{\mathrm{Z}}\right)$ determinations. Results obtained with further PDF sets constitute the PDFset uncertainty as defined previously. They are listed in Tables 4 and 7 together with the

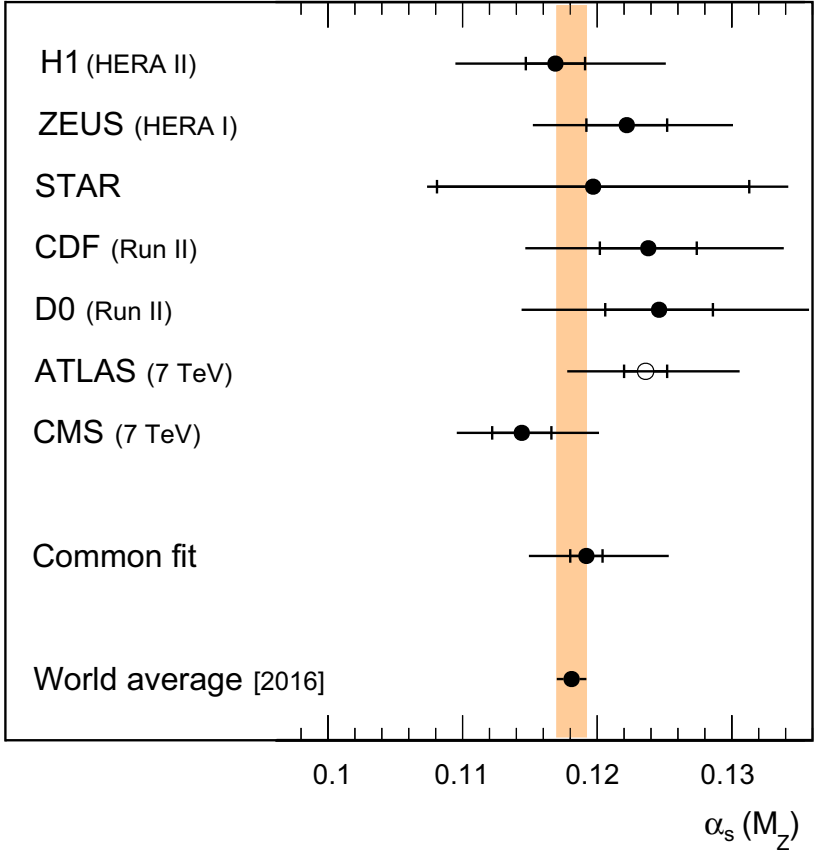

Fig. 4 The $\alpha_{\mathrm{S}}\left(M_{\mathrm{Z}}\right)$ values from fits to individual data sets are compared to our simultaneous fit to H1, ZEUS, STAR, CDF, D0, and CMS data, and to the world average value [1]. The inner error bars represent the experimental uncertainty and the outer ones the total uncertainty. For reasons explained in the text, the ATLAS data are excluded from the common fit and only the result of a separate fit is indicated by the open circle

$\mathrm{PDF}^{2}$ and $\mathrm{PDF} \alpha_{\mathrm{s}}$ uncertainties as appropriate for the respective PDF set. The values of $\chi^{2} / n_{\text {dof }}$ for the main PDF sets can be read off from row six of Fig. 3 right.

The $\alpha_{\mathrm{s}}\left(M_{\mathrm{Z}}\right)$ values from fits using the various PDF sets given in Table 4 are found to be consistent within the experimental uncertainty. The NP, PDF, and $\mathrm{PDF} \alpha_{\mathrm{s}}$ uncertainties are smaller than the experimental uncertainty, while the PDFset uncertainty is of a similar size as the experimental one. The scale uncertainty is the largest individual uncer-

${ }^{2}$ For HERAPDF2.0, the PDF uncertainty does not include the "model" or "parameterisation" uncertainties as those are represented here by the PDFset uncertainty. 
Fig. 5 Ratio of data over theory for the selected inclusive jet cross sections listed in Table 2 as a function of jet $p_{\mathrm{T}}$. The NLO predictions are computed with the NNPDF3.0 PDF set for the fitted $\alpha_{\mathrm{S}}\left(M_{\mathrm{Z}}\right)$ value of 0.1192 , which is determined considering all presented data except for the ATLAS data set. They are complemented with non-perturbative corrections and, where appropriate, with electroweak corrections

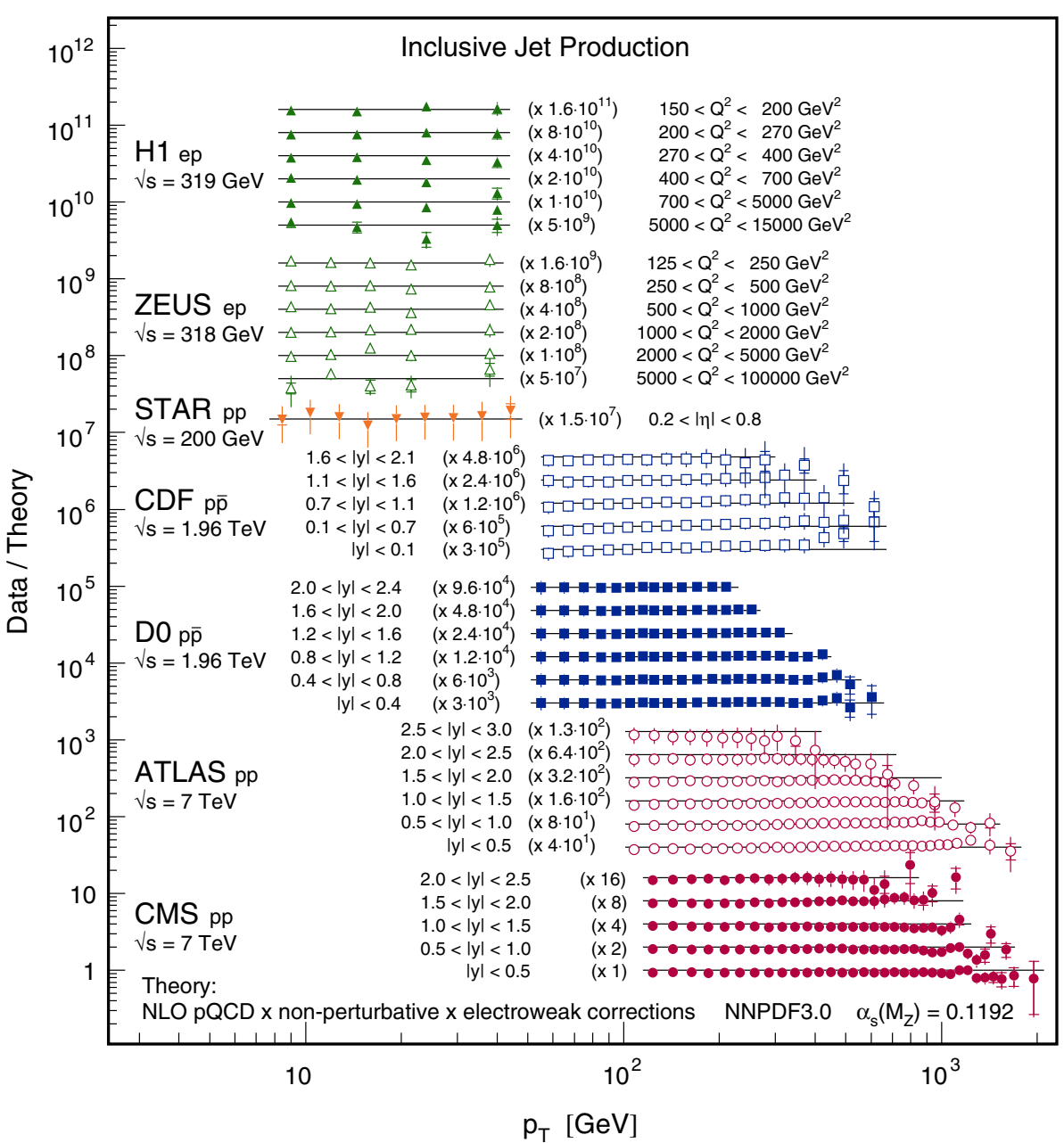

tainty and is more than three times larger than any other uncertainty. Results of the $\alpha_{\mathrm{S}}\left(M_{\mathrm{Z}}\right)$ extractions from single data sets, cf. Appendix B, from the simultaneous $\alpha_{\mathrm{S}}\left(M_{\mathrm{Z}}\right)$ extraction from all data sets, and the world average [1] are compared in Fig. 4 and are seen to be consistent with each other.

The ratio of data to the predictions as a function of jet $p_{\mathrm{T}}$ for all selected data sets is presented in Fig. 5. The predictions are computed for $\alpha_{\mathrm{S}}\left(M_{\mathrm{Z}}\right)=0.1192$ as obtained in this analysis. Visually, all data sets are well described by the theory predictions.

\section{Summary and outlook}

Inclusive jet cross section data from different experiments at various particle colliders with jet transverse momenta ranging from $7 \mathrm{GeV}$ up to $2 \mathrm{TeV}$ are explored for determinations of $\alpha_{\mathrm{s}}\left(M_{\mathrm{Z}}\right)$ using next-to-leading order predictions.

Previous $\alpha_{\mathrm{S}}\left(M_{\mathrm{Z}}\right)$ determinations reported by the CMS, D0, and H1 collaborations [27,61,62] are taken as a baseline, and these $\alpha_{\mathrm{S}}\left(M_{\mathrm{Z}}\right)$ extraction methods, which differ in various aspects, are applied to inclusive jet cross section data measured by the ATLAS, CDF, CMS, D0, H1, STAR, and ZEUS experiments [16,20,27,32,36,41,62]. Differences among the $\alpha_{\mathrm{S}}\left(M_{\mathrm{Z}}\right)$ results due to the extraction technique are found to be negligible in most cases. A new extraction method is proposed, which combines aspects of the baseline approaches above.

In a statistical analysis, data measured by the CDF, CMS, D0, H1, STAR, and ZEUS experiments are found to be well described by pQCD predictions at next-to-leading order, and hence are considered to be mutually consistent. Moreover, the values of $\alpha_{\mathrm{S}}\left(M_{\mathrm{Z}}\right)$ determined from each individual data set are found to be consistent among each other. By determining $\alpha_{\mathrm{S}}\left(M_{\mathrm{Z}}\right)$ simultaneously from these data, the experimental uncertainty of $\alpha_{\mathrm{S}}\left(M_{\mathrm{Z}}\right)$ is reduced to $1.0 \%$, as compared to $1.9 \%$ when only the single most precise data set of that selection is considered.

The largest contribution to the uncertainty of $\alpha_{\mathrm{S}}\left(M_{\mathrm{Z}}\right)$ originates from the renormalisation scale dependence of the next-to-leading order pQCD calculation. This uncertainty is expected to be reduced once the next-to-next-to-leading order predictions become available for such studies. Furthermore, 
a reevaluation of the non-perturbative corrections and their uncertainties for all data sets in a consistent manner is recommended for a determination of $\alpha_{\mathrm{S}}\left(M_{\mathrm{Z}}\right)$ at high precision. The presented study and the developed analysis framework provide a solid basis for future determinations of $\alpha_{\mathrm{S}}\left(M_{\mathrm{Z}}\right)$ and facilitate the inclusion of additional data sets, further observables, and improved theory predictions.

Acknowledgements We thank our colleagues in the CMS, D0, and H1 collaborations for fruitful discussions, and K. Bjørke and D. Reichelt for early related studies. K. Rabbertz thanks G. Flouris, P. Kokkas, and the colleagues from the PROSA Collaboration. D. Savoiu acknowledges the support by the DFG-funded Doctoral School "Karlsruhe School of Elementary and Astroparticle Physics: Science and Technology". M. Wobisch also wishes to thank the Louisiana Board of Regents Support Fund for the support through the Eva J. Cunningham Endowed Professorship.

Data Availability Statement This manuscript has associated data in a data repository. [Authors' comment: All analyzed data have been published by the respective collaborations and are available online on HEPData.]

Open Access This article is distributed under the terms of the Creative Commons Attribution 4.0 International License (http://creativecomm ons.org/licenses/by/4.0/), which permits unrestricted use, distribution, and reproduction in any medium, provided you give appropriate credit to the original author(s) and the source, provide a link to the Creative Commons license, and indicate if changes were made.

Funded by SCOAP . $^{3}$.

\section{A Definition of the $\chi^{2}$ expression for the common-type method}

In the common-type method the $\chi^{2}$ expression, which is subject to the minimisation algorithm, is defined as

$$
\begin{aligned}
\chi^{2}= & \sum_{i j}\left(\log \frac{m_{i}}{t_{i}}\right)\left[\left(\mathcal{V}_{\mathrm{exp}}+\mathcal{V}_{\mathrm{PDF}}+\mathcal{V}_{\mathrm{NP}}\right)^{-1}\right]_{i j} \\
& \left(\log \frac{m_{j}}{t_{j}}\right),
\end{aligned}
$$

where the sum runs over all data points $i$ and $j$ of the measured cross sections $m_{i}, m_{j}$ and theory predictions $t_{i}, t_{j}$. The covariance matrices $\mathcal{V}$ represent the relative experimental, PDF, and NP uncertainties. A similar $\chi^{2}$ definition, taking into account only experimental uncertainties, was employed by the H1 Collaboration $[5,27,28]$. For the calculation of the covariance matrices, all uncertainties are symmetrised, if necessary, by averaging the corresponding "up" and "down" shifts in $\alpha_{\mathrm{S}}\left(M_{\mathrm{Z}}\right)$ in quadrature while keeping the sign of bin-to-bin correlations. Uncertainty contributions to the total covariance matrix that are fully correlated across all observable bins in Eq. (4) can alternatively be expressed in an equivalent form with nuisance parameters.
B Common-type extraction of $\alpha_{\mathrm{S}}\left(M_{\mathrm{Z}}\right)$ from single inclusive jet data sets

Detailed results of the common-type method applied to the individual data sets are given in Table 5. The result for the H1 data agrees with the value published in Ref. [27]. Even though using the full D0 data set with 110 points, the extracted $\alpha_{\mathrm{S}}\left(M_{\mathrm{Z}}\right)$ value is consistent with the value achieved by the D0 Collaboration at NLO for a subset of 22 points in Ref. [61]. For the CMS measurement, the common-type method leads to a consistent but somewhat lower result than reported in Ref. [62] for various PDFs. Our result for the ZEUS data is compatible with the value obtained by the ZEUS Collaboration from a single-differential variant of the measurement in a reduced phase space as published in Ref. [33]. With respect to the ATLAS, CDF, and STAR inclusive jet data, this study constitutes the first $\alpha_{\mathrm{S}}\left(M_{\mathrm{Z}}\right)$ determination from either data set. Within uncertainties, all $\alpha_{\mathrm{S}}\left(M_{\mathrm{Z}}\right)$ values are consistent with each other and with the world average.

The individual uncertainties compare as follows:

- The experimental uncertainty of $\alpha_{\mathrm{s}}\left(M_{\mathrm{Z}}\right)$ is roughly comparable between experiments at the same collider. It is largest for the STAR data, and smallest for the ATLAS data.

- The NP uncertainties are found to vary significantly, even between data sets in similar kinematic regions, for instance between CDF and D0 . In case of the LHC experiments the NP uncertainties appear to be negligible.

- The PDF uncertainty as estimated with the NNPDF3.0 PDF set is smaller than the experimental uncertainty. For the HERA data, the PDF uncertainty is found to be moderately smaller than for Tevatron or LHC data as observed also with other PDF sets.

- For all data sets, the PDF $\alpha_{\mathrm{s}}$ uncertainty is rather small. This observation justifies to neglect the $\alpha_{\mathrm{S}}$ dependence of the PDFs in the $\alpha_{\mathrm{s}}\left(M_{\mathrm{Z}}\right)$ determinations and to assign a separately derived uncertainty instead.

- The PDFset uncertainty constitutes the largest contribution of the PDF related ones.

- The largely dominating scale uncertainty is of similar size in case of HERA and LHC data and somewhat larger for Tevatron data or the STAR experiment.

To estimate the effect of varying theoretical techniques or input data compositions among the available PDF sets, we present the results of $\alpha_{\mathrm{s}}\left(M_{\mathrm{Z}}\right)$ determinations from single measurements in columns $2-5$ of Tables 6 and 7. For the first Table 6 the alternative PDF sets ABMP16, CT14, HERAPDF2.0, and MMHT2014 are selected in addition to our base choice of NNPDF3.0. Out of these the ABMP16 and HERAPDF2.0 sets do not use any jet data, while the other three do include some but not all of the inves- 
Table 5 Results of common-type $\alpha_{\mathrm{S}}\left(M_{\mathrm{Z}}\right)$ extractions from individual inclusive jet data sets using the NNPDF3.0 PDF set. The values for $\alpha_{\mathrm{s}}\left(M_{\mathrm{Z}}\right)$ are provided along with the experimental and theoretical uncertainties. The latter consist of contributions originating from NP effects, the propagation of the PDF uncertainties, the choices of the PDF
$\alpha_{\mathrm{s}}\left(M_{\mathrm{Z}}\right)$ value and the PDF set, and the scale uncertainty. The quadratic sum of the experimental and theoretical uncertainties is quoted as the total uncertainty. The corresponding $\chi^{2} / n_{\text {dof }}$ values are displayed in column five of Fig. 3left

\begin{tabular}{|c|c|c|c|c|c|c|c|c|c|}
\hline \multirow[t]{2}{*}{ Data set } & \multirow[t]{2}{*}{$\alpha_{\mathrm{S}}\left(M_{\mathrm{Z}}\right)$} & \multicolumn{8}{|c|}{ Uncertainties (scaled by factor $10^{4}$ ) } \\
\hline & & Exp & NP & PDF & $\mathrm{PDF} \alpha_{\mathrm{s}}$ & PDFset & Scale & Theo & Total \\
\hline H1 & 0.1169 & 22 & 9 & 8 & 4 & 7 & $\begin{array}{l}+58 \\
-47\end{array}$ & $\begin{array}{l}+60 \\
-49\end{array}$ & $\begin{array}{l}+64 \\
-54\end{array}$ \\
\hline ZEUS & 0.1222 & 30 & 18 & 9 & 3 & 12 & $\begin{array}{l}+48 \\
-33\end{array}$ & $\begin{array}{l}+54 \\
-40\end{array}$ & $\begin{array}{l}+62 \\
-50\end{array}$ \\
\hline STAR & 0.1197 & 116 & - & 50 & 26 & 106 & $\begin{array}{l}+87 \\
{ }_{-41}\end{array}$ & $\begin{array}{l}+148 \\
-127\end{array}$ & $\begin{array}{l}+188 \\
{ }_{-172}^{+17}\end{array}$ \\
\hline $\mathrm{CDF}$ & 0.1238 & 36 & 13 & 14 & 9 & 58 & $\begin{array}{l}+83 \\
-39\end{array}$ & $\begin{array}{l}+104 \\
-73\end{array}$ & $\begin{array}{l}+110 \\
-81\end{array}$ \\
\hline D0 & 0.1246 & 40 & 23 & 21 & 8 & 62 & $\begin{array}{l}+104 \\
-76\end{array}$ & $\begin{array}{l}+125 \\
-103\end{array}$ & $\begin{array}{l}+131 \\
-111\end{array}$ \\
\hline ATLAS & 0.1236 & 16 & 3 & 15 & 8 & 30 & $\begin{array}{l}+65 \\
-34\end{array}$ & $\begin{array}{l}+74 \\
-49\end{array}$ & $\begin{array}{l}+76 \\
-51\end{array}$ \\
\hline CMS & 0.1144 & 22 & 1 & 14 & 9 & 24 & $\begin{array}{l}+58 \\
-24\end{array}$ & $\begin{array}{l}+65 \\
-38\end{array}$ & $\begin{array}{r}+69 \\
-44\end{array}$ \\
\hline
\end{tabular}

Table 6 Results of common-type fits to single inclusive jet data sets for varying PDF sets. Listed are the $\alpha_{\mathrm{s}}\left(M_{\mathrm{Z}}\right)$ results and the respective PDF and $\mathrm{PDF} \alpha_{\mathrm{S}}$ uncertainty. For the purpose of a more compact presentation, the employed PDF sets ABMP16 (AB), CT14 (CT), HERAPDF2.0
(H2), MMHT2014 (MM), and NNPDF3.0 (NN) are abbreviated here to the two-letter acronyms given in parentheses. The results for NNPDF3.0 are listed for completeness and are identical to the ones displayed in Table 5 . Uncertainties are scaled by a factor of $10^{4}$

\begin{tabular}{|c|c|c|c|c|c|c|c|c|c|c|c|c|c|c|c|}
\hline \multirow[t]{2}{*}{ Data set } & \multicolumn{5}{|l|}{$\alpha_{\mathrm{S}}\left(M_{\mathrm{Z}}\right)$} & \multicolumn{5}{|c|}{$\begin{array}{l}\text { PDF uncertainty (scaled } \\
\text { by factor } 10^{4} \text { ) }\end{array}$} & \multicolumn{5}{|c|}{$\begin{array}{l}\operatorname{PDF} \alpha_{\mathrm{s}} \text { uncertainty } \\
\left(\text { scaled by factor } 10^{4}\right)\end{array}$} \\
\hline & $\mathrm{AB}$ & $\mathrm{CT}$ & $\mathrm{H} 2$ & MM & NN & $\mathrm{AB}$ & CT & $\mathrm{H} 2$ & $\mathrm{MM}$ & $\mathrm{NN}$ & $\mathrm{AB}$ & $\mathrm{CT}$ & $\mathrm{H} 2$ & MM & $\mathrm{NN}$ \\
\hline $\mathrm{H} 1$ & 0.1155 & 0.1169 & 0.1150 & 0.1168 & 0.1169 & 4 & 11 & 3 & 7 & 8 & 9 & 3 & 8 & 4 & 4 \\
\hline ZEUS & 0.1203 & 0.1228 & 0.1192 & 0.1224 & 0.1222 & 5 & 11 & 4 & 7 & 9 & 9 & 1 & 8 & 2 & 3 \\
\hline STAR & 0.1034 & 0.1232 & 0.1129 & 0.1159 & 0.1197 & 22 & 63 & 30 & 37 & 50 & 18 & 5 & 1 & 12 & 26 \\
\hline $\mathrm{CDF}$ & 0.1303 & 0.1239 & 0.1329 & 0.1243 & 0.1238 & 13 & 29 & 8 & 19 & 14 & 27 & 1 & 17 & 1 & 9 \\
\hline D0 & 0.1344 & 0.1221 & 0.1289 & 0.1237 & 0.1246 & 19 & 34 & 16 & 23 & 21 & 29 & 2 & 11 & 0 & 8 \\
\hline ATLAS & 0.1263 & 0.1211 & 0.1211 & 0.1203 & 0.1236 & 13 & 22 & 13 & 17 & 15 & 11 & 0 & 6 & 3 & 8 \\
\hline CMS & 0.1186 & 0.1165 & 0.1146 & 0.1154 & 0.1144 & 10 & 24 & 14 & 19 & 14 & 14 & 2 & 6 & 2 & 9 \\
\hline
\end{tabular}

Table 7 Results of common-type fits to single inclusive jet data sets and to all data sets together except ATLAS (Common) for varying PDFs. Listed are the $\alpha_{\mathrm{S}}\left(M_{\mathrm{Z}}\right)$ results and the respective PDF and PDF $\alpha_{\mathrm{S}}$ uncertainty. For the purpose of a more compact presentation, the employed
NNPDF3.0 variants NNPDF3.0_hera (NH), NNPDF3.0_atlas (NA), NNPDF3.0_cms (NC), NNPDF3.0_nolhc (NL), and NNPDF3.0_nojet (NJ) are abbreviated here to the two-letter acronyms given in parentheses. Uncertainties are scaled by a factor of $10^{4}$

\begin{tabular}{|c|c|c|c|c|c|c|c|c|c|c|c|c|c|c|c|}
\hline \multirow[t]{2}{*}{ Data set } & \multicolumn{5}{|l|}{$\alpha_{\mathrm{s}}\left(M_{\mathrm{Z}}\right)$} & \multicolumn{5}{|c|}{$\begin{array}{l}\text { PDF uncertainty (scaled } \\
\text { by factor } 10^{4} \text { ) }\end{array}$} & \multicolumn{5}{|c|}{$\begin{array}{l}\mathrm{PDF} \alpha_{\mathrm{S}} \text { uncertainty } \\
\left(\text { scaled by factor } 10^{4}\right)\end{array}$} \\
\hline & $\mathrm{NH}$ & NA & $\mathrm{NC}$ & NL & $\mathrm{NJ}$ & $\mathrm{NH}$ & NA & $\mathrm{NC}$ & $\mathrm{NL}$ & $\mathrm{NJ}$ & $\mathrm{NH}$ & NA & $\mathrm{NC}$ & NL & $\mathrm{NJ}$ \\
\hline H1 & 0.1159 & 0.1172 & 0.1177 & 0.1168 & 0.1160 & 15 & 10 & 9 & 8 & 13 & 11 & 5 & 6 & 6 & 12 \\
\hline ZEUS & 0.1235 & 0.1224 & 0.1229 & 0.1224 & 0.1217 & 21 & 11 & 11 & 10 & 16 & 12 & 4 & 5 & 4 & 11 \\
\hline STAR & 0.1312 & 0.1224 & 0.1306 & 0.1234 & 0.1247 & 124 & 74 & 79 & 75 & 95 & 18 & 20 & 14 & 32 & 23 \\
\hline $\mathrm{CDF}$ & 0.1212 & 0.1266 & 0.1260 & 0.1232 & 0.1187 & 55 & 25 & 27 & 22 & 28 & 8 & 4 & 8 & 6 & 7 \\
\hline D0 & 0.1240 & 0.1277 & 0.1239 & 0.1239 & 0.1241 & 53 & 32 & 32 & 30 & 37 & 2 & 4 & 5 & 4 & 6 \\
\hline ATLAS & 0.1238 & 0.1255 & 0.1232 & 0.1229 & 0.1211 & 30 & 24 & 24 & 21 & 23 & 8 & 2 & 4 & 5 & 11 \\
\hline CMS & 0.1170 & 0.1171 & 0.1187 & 0.1140 & 0.1137 & 33 & 24 & 26 & 21 & 24 & 8 & 4 & 6 & 6 & 12 \\
\hline Common & 0.1206 & 0.1205 & 0.1209 & 0.1194 & 0.1196 & 13 & 10 & 10 & 9 & 12 & 8 & 4 & 4 & 4 & 9 \\
\hline
\end{tabular}

tigated jet data among their input data. In Table 7 the five NNPDF3.0 variants NNPDF3.0_hera, NNPDF3.0_atlas, NNPDF3.0_cms, NNPDF3.0_nolhc, and NNPDF3.0_nojet are used. In contrast to the base set NNPDF3.0, the first three exclusively evaluate HERA data, HERA and ATLAS data, and HERA and CMS data, while NNPDF3.0_nolhc 


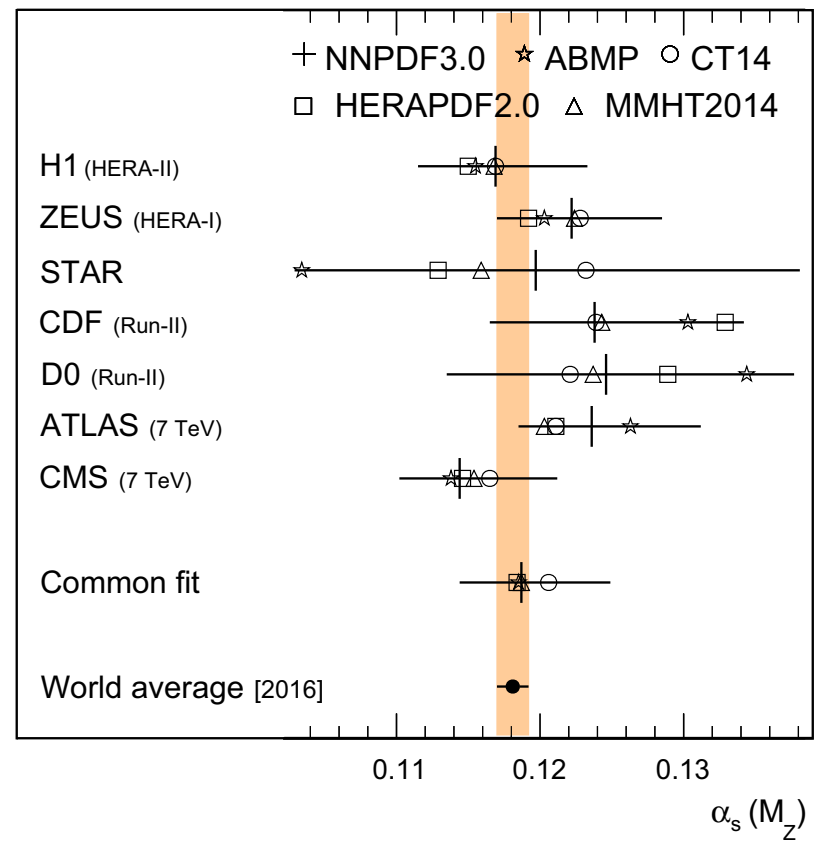

Fig. 6 Comparison of results for $\alpha_{\mathrm{S}}\left(M_{\mathrm{Z}}\right)$ obtained with the alternative PDF sets ABMP16, CT14, HERAPDF2.0, and MMHT2014 (left), and (right) with the NNPDF3.0 variants NNPDF3.0_hera (NH), NNPDF3.0_atlas (NA), NNPDF3.0_cms (NC), NNPDF3.0_nolhc

and NNPDF3.0_nojet exclude all LHC respectively all jet data from the PDF determination. The further columns in Tables 6 and 7 present the PDF and $\mathrm{PDF} \alpha_{\mathrm{S}}$ uncertainty for the respective PDF sets.

The spread among the $\alpha_{\mathrm{S}}\left(M_{\mathrm{Z}}\right)$ determinations from a single data set with varying PDF sets is illustrated in the two plots of Fig. 6. For each of the individual data sets, the results are mostly consistent. Larger deviations are observed for the Tevatron data when using the ABMP16 and HERAPDF2.0 sets, and for the STAR data in conjunction with the ABMP16 or NNPDF3.0_hera and NNPDF3.0_cms PDF sets. Interestingly, some of the largest differences appear between the results for Tevatron and LHC jet data with the PDF sets ABMP16 and NNPDF3.0_nojet that both explicitly exclude jets from the PDF extraction.

The PDF uncertainty obtained with different PDF sets for the same data set is largest for CT14 and smallest for HERAPDF2.0, for which "model" or "parameterisation" uncertainties have not been included. This may explain the significantly larger PDF uncertainties for the NNPDF3.0_hera set as compared to HERAPDF2.0. It is interesting to note that on the one hand side the NNPDF3.0_nolhc PDF set leads to smaller uncertainties than the one without jet data, NNPDF3.0_nojet, but on the other hand the ABMP16 PDF uncertainties come out significantly smaller than both.

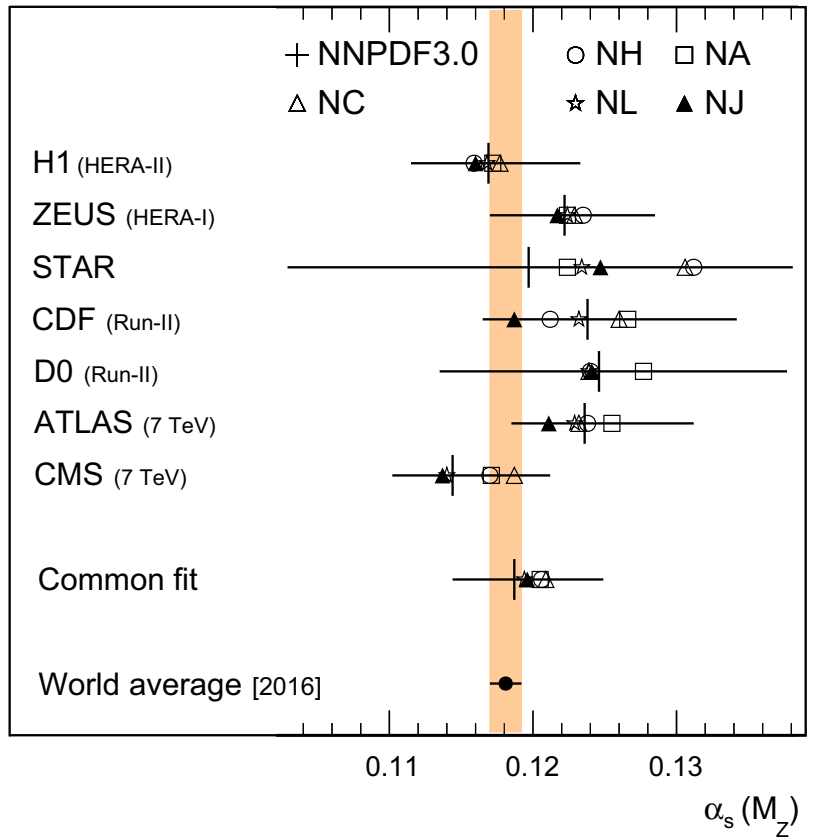

(NL), and NNPDF3.0_nojet (NJ). The values are compared to the value of $\alpha_{\mathrm{S}}\left(M_{\mathrm{Z}}\right)$ obtained with the NNPDF3.0 set and to the world average value [1]. The horizontal error bars, attached to the points representing the NNPDF3.0 results, indicate the total uncertainty

With respect to the $\mathrm{PDF} \alpha_{\mathrm{s}}$ uncertainties we observe that, not surprisingly, PDF sets without jet data, ABMP16, HERAPDF2.0, NNPDF3.0_hera, and NNPDF3.0_nojet, tend to give significantly larger $\mathrm{PDF} \alpha_{\mathrm{S}}$ uncertainties than any other PDF set. The CT14 or MMHT2014 PDFs in particular exhibit rather small $\mathrm{PDF} \alpha_{\mathrm{s}}$ uncertainties.

Clearly, one reason for the observed pattern of $\alpha_{\mathrm{S}}\left(M_{\mathrm{Z}}\right)$ values and uncertainties lies in the different selections of data considered for the PDF determination. The NNPDF3.0 set variants have proven to be very useful to acquire some insight into the possible impact of correlations on the presented $\alpha_{\mathrm{S}}\left(M_{\mathrm{Z}}\right)$ determinations and it is desirable to have similar possibilities for the other PDF sets. The spread among $\alpha_{\mathrm{S}}\left(M_{\mathrm{Z}}\right)$ results using various PDF sets, however, is much larger than could potentially be explained by the inclusion of some jet data twice, first in the PDF fit and secondly in our $\alpha_{\mathrm{S}}\left(M_{\mathrm{Z}}\right)$ determination. Therefore we conclude that for the presented study the effect of correlations between jet data in a PDF set and our $\alpha_{\mathrm{S}}\left(M_{\mathrm{Z}}\right)$ determination is rather small compared to other effects and is covered by the PDFset uncertainty defined as follows:

Half of the envelope constructed from the five values for the ABMP16, CT14, MMHT2014, and NNPDF3.0_nojet PDFs together with the NNPDF3.0 result constitutes the PDFset uncertainty shown in column seven of Table 5. These sets 
either comprise a maximum of data other than jet cross sections or no jet cross sections at all.

\section{References}

1. C. Patrignani, et al. (Particle Data Group), Review of particle physics. Chin. Phys. C 40, 100001 (2016). https://doi.org/10. 1088/1674-1137/40/10/100001

2. G. Dissertori, The determination of the strong coupling constant. Adv. Ser. Direct. High Energy Phys. 26, 113 (2016). https://doi. org/10.1142/9789814733519_0006. arXiv:1506.05407

3. D. d'Enterria, P. Skands, eds., Proceedings of the Workshop on High-Precision $\alpha_{s}$ Measurements: From LHC to FCC-ee, October 12-13, 2015. Geneva, Switzerland (2015). arXiv:1512.05194

4. K. Rabbertz, Jet Physics at the LHC, 1st edn., Springer Tracts in Modern Physics (Springer, Berlin, 2016)

5. H1 Collaboration, Determination of the strong coupling constant $\alpha_{S}\left(M_{Z}\right)$ in next-to-next-to-leading order QCD using $\mathrm{H} 1$ jet cross section measurements. Eur. Phys. J. C 77(11), 791 (2017). https:// doi.org/10.1140/epjc/s10052-017-5314-7. arXiv:1709.07251

6. J. Currie, E.W.N. Glover, J. Pires, Next-to-next-to leading order QCD predictions for single jet inclusive production at the LHC. Phys. Rev. Lett. 118, 072002 (2017). https://doi.org/10.1103/ PhysRevLett.118.072002. arXiv: 1611.01460

7. J. Currie, T. Gehrmann, A. Huss, J. Niehues, NNLO QCD corrections to jet production in deep inelastic scattering. JHEP 07, 018 (2017). https://doi.org/10.1007/JHEP07(2017)018. arXiv: 1703.05977

8. UA2 Collaboration, Observation of very large transverse momentum jets at the CERN $\bar{p} p$ collider. Phys. Lett. B 118, 203 (1982). https://doi.org/10.1016/0370-2693(82)90629-3

9. UA1 Collaboration, Hadronic jet production at the CERN protonantiproton collider. Phys. Lett. B 132, 214 (1983). https://doi.org/ 10.1016/0370-2693(83)90254-X

10. UA1 Collaboration, Measurement of the inclusive jet crosssection at the CERN p anti-p collider. Phys. Lett. B 172, 461 (1986). https://doi.org/10.1016/0370-2693(86)90290-X

11. UA2 Collaboration, Measurement of the $\sqrt{(} s)$ dependence of jet production at the CERN anti- $p$ p collider. Phys. Lett. B 160, 349 (1985). https://doi.org/10.1016/0370-2693(85)91341-3

12. CDF Collaboration, Comparison of jet production in $\bar{p} p$ collisions at $\sqrt{s}=546 \mathrm{GeV}$ and $1800 \mathrm{GeV}$. Phys. Rev. Lett. 70, 1376 (1993). https://doi.org/10.1103/PhysRevLett.70.1376

13. CDF Collaboration, Measurement of the inclusive jet cross section in $\bar{p} p$ collisions at $\sqrt{s}=1.8 \mathrm{TeV}$. Phys. Rev. D 64, 032001 (2001). https://doi.org/10.1103/PhysRevD.65.039903, https:// doi.org/10.1103/PhysRevD.64.032001. arXiv:hep-ph/0102074

14. CDF Collaboration, Measurement of the inclusive jet cross section in $p \bar{p}$ interactions at $\sqrt{s}=1.96-\mathrm{TeV}$ using a cone-based jet algorithm. Phys. Rev. D 74, 071103 (2006). https://doi.org/10. 1103/PhysRevD.74.071103. arXiv:hep-ex/0512020

15. CDF Collaboration, Measurement of the inclusive jet cross section using the $k(t)$ algorithm in $\mathrm{p}$ anti-p collisions at $\sqrt{s}=1.96-$ TeV. Phys. Rev. Lett. 96, 122001 (2006). https://doi.org/10.1103/ PhysRevLett.96.122001. arXiv:hep-ex/0512062

16. CDF Collaboration, Measurement of the inclusive jet cross section using the $k_{\mathrm{T}}$ algorithm in $p \bar{p}$ collisions at $\sqrt{s}=1.96 \mathrm{TeV}$ with the CDF II detector. Phys. Rev. D 75, 092006 (2007). https:// doi.org/10.1103/PhysRevD.75.119901, https://doi.org/10.1103/ PhysRevD.75.092006. arXiv:hep-ex/0701051

17. CDF Collaboration, Measurement of the inclusive jet cross section at the fermilab tevatron $p \bar{p}$ collider using a cone-based jet algorithm. Phys. Rev. D 78, 052006 (2008). https://doi.org/10.
1103/PhysRevD.79.119902, https://doi.org/10.1103/PhysRevD. 78.052006. arXiv:0807.2204

18. D0 Collaboration, Inclusive jet production in $p \bar{p}$ collisions. Phys. Rev. Lett. 86, 1707 (2001). https://doi.org/10.1103/PhysRevLett. 86.1707. arXiv:hep-ex/0011036

19. D0 Collaboration, High- $p_{T}$ jets in $\bar{p} p$ collisions at $\sqrt{s}=630$ GeV and 1800 GeV. Phys. Rev. D 64, 032003 (2001). https://doi. org/10.1103/PhysRevD.64.032003. arXiv:hep-ex/0012046

20. D0 Collaboration, Measurement of the inclusive jet crosssection in $p \bar{p}$ collisions at $\sqrt{s}=1.96 \mathrm{TeV}$. Phys. Rev. Lett. 101, 062001 (2008). https://doi.org/10.1103/PhysRevLett.101. 062001. arXiv:0802.2400

21. H1 Collaboration, Measurement and QCD analysis of jet crosssections in deep-inelastic positron-proton collisions at $\sqrt{s}$ of 300 GeV. Eur. Phys. J. C 19, 289 (2001). https://doi.org/10.1007/ s100520100621. arXiv:hep-ex/0010054

22. H1 Collaboration, Measurement of inclusive jet cross-sections in deep inelastic ep scattering at HERA. Phys. Lett. B 542, 193 (2002). https://doi.org/10.1016/S0370-2693(02)02375-4. arXiv:hep-ex/0206029

23. H1 Collaboration, Measurement of inclusive jet cross-sections in photoproduction at HERA. Eur. Phys. J. C 29, 497 (2003). https:// doi.org/10.1140/epjc/s2003-01262-9. arXiv:hep-ex/0302034

24. H1 Collaboration, Measurement of inclusive jet production in deep-inelastic scattering at high $Q^{2}$ and determination of the strong coupling. Phys. Lett. B 653, 134 (2007). https://doi.org/ 10.1016/j.physletb.2007.07.050. arXiv:0706.3722

25. H1 Collaboration, Jet production in ep collisions at High $Q^{2}$ and determination of $\alpha_{s}$. Eur. Phys. J. C 65, 363 (2010). https://doi. org/10.1140/epjc/s10052-009-1208-7. arXiv:0904.3870

26. H1 Collaboration, Jet production in ep collisions at Low $Q^{2}$ and determination of $\alpha_{s}$. Eur. Phys. J. C 67, 1 (2010). https://doi.org/ 10.1140/epjc/s10052-010-1282-x. arXiv:0911.5678

27. H1 Collaboration, Measurement of multijet production in $e p$ collisions at high $Q^{2}$ and determination of the strong coupling $\alpha_{s}$. Eur. Phys. J. C 75, 65 (2015). https://doi.org/10.1140/epjc/ s10052-014-3223-6. arXiv:1406.4709

28. H1 Collaboration, Measurement of jet production cross sections in deep-inelastic ep scattering at HERA. Eur. Phys. J. C 77(4), 215 (2017). https://doi.org/10.1140/epjc/s10052-017-4717-9. arXiv: 1611.03421

29. ZEUS Collaboration, High- $E_{T}$ inclusive jet cross sections in photoproduction at HERA. Eur. Phys. J. C 4, 591 (1998). https://doi. org/10.1007/s100529800916. arXiv:hep-ex/9802012

30. ZEUS Collaboration, Inclusive jet cross sections in the Breit frame in neutral current deep inelastic scattering at HERA and determination of $\alpha_{s}$. Phys. Lett. B 547, 164 (2002). https://doi.org/10. 1016/S0370-2693(02)02763-6. arXiv:hep-ex/0208037

31. ZEUS Collaboration, Scaling violations and determination of $\alpha_{s}$ from jet production in gamma $\mathrm{p}$ interactions at HERA. Phys. Lett. B 560, 7 (2003). https://doi.org/10.1016/ S0370-2693(03)00380-0. arXiv:hep-ex/0212064

32. ZEUS Collaboration, Inclusive-jet and dijet cross sections in deep inelastic scattering at HERA. Nucl. Phys. B 765, 1 (2007). https://doi.org/10.1016/j.nuclphysb.2006.09.018. arXiv:hep-ex/0608048

33. ZEUS Collaboration, Jet-radius dependence of inclusive-jet cross sections in deep inelastic scattering at HERA. Phys. Lett. B 649, 12 (2007). https://doi.org/10.1016/j.physletb.2007.03.039. arXiv:hep-ex/0701039

34. ZEUS Collaboration, Inclusive-jet cross sections in NC DIS at HERA and a comparison of the kT, anti-kT and SIScone jet algorithms. Phys. Lett. B 691, 127 (2010). https://doi.org/10.1016/j. physletb.2010.06.015. arXiv:1003.2923 
35. ZEUS Collaboration, Inclusive-jet photoproduction at HERA and determination of $\alpha_{s}$. Nucl. Phys. B 864, 1 (2012). https://doi.org/ 10.1016/j.nuclphysb.2012.06.006. arXiv:1205.6153

36. STAR Collaboration, Longitudinal double-spin asymmetry and cross section for inclusive jet production in polarized proton collisions at $\sqrt{s}=200 \mathrm{GeV}$. Phys. Rev. Lett. 97, 252001 (2006). https://doi.org/10.1103/PhysRevLett.97.252001. arXiv:hep-ex/0608030

37. ALICE Collaboration, Measurement of the inclusive differential jet cross section in $p p$ collisions at $\sqrt{s}=2.76 \mathrm{TeV}$. Phys. Lett. B 722, 262 (2013). https://doi.org/10.1016/j.physletb.2013.04.026. arXiv: 1301.3475

38. ATLAS Collaboration, Measurement of inclusive jet and dijet cross sections in proton-proton collisions at $7 \mathrm{TeV}$ centre-ofmass energy with the ATLAS detector. Eur. Phys. J. C 71, 1512 (2011). https://doi.org/10.1140/epjc/s10052-010-1512-2. arXiv: 1009.5908

39. ATLAS Collaboration, Measurement of inclusive jet and dijet production in $p p$ collisions at $\sqrt{s}=7 \mathrm{TeV}$ using the ATLAS detector. Phys. Rev. D 86, 014022 (2012). https://doi.org/10.1103/ PhysRevD.86.014022. arXiv:1112.6297

40. ATLAS Collaboration, Measurement of the inclusive jet cross section in $p p$ collisions at $\sqrt{s}=2.76 \mathrm{TeV}$ and comparison to the inclusive jet cross section at $\sqrt{s}=7 \mathrm{TeV}$ using the ATLAS detector. Eur. Phys. J. C 73, 2509 (2013). https://doi.org/10.1140/ epjc/s10052-013-2509-4. arXiv:1304.4739

41. ATLAS Collaboration, Measurement of the inclusive jet crosssection in proton-proton collisions at $\sqrt{s}=7 \mathrm{TeV}$ using $4.5 \mathrm{fb}^{-1}$ of data with the ATLAS detector. JHEP $\mathbf{0 2}$, 153 (2015). https://doi.org/10.1007/JHEP02(2015)153, https:// doi.org/10.1007/JHEP09(2015)141. arXiv:1410.8857. [Erratum: JHEP 09, 141 (2015)]

42. ATLAS Collaboration, Measurement of the inclusive jet crosssections in proton-proton collisions at $\sqrt{s}=8 \mathrm{TeV}$ with the ATLAS detector. JHEP 09, 020 (2017). https://doi.org/10.1007/ JHEP09(2017)020. arXiv:1706.03192

43. ATLAS Collaboration, Jet energy scale measurements and their systematic uncertainties in proton-proton collisions at $\sqrt{s}=13 \mathrm{TeV}$ with the ATLAS detector. Phys. Rev. D 96(7), 072002 (2017). https://doi.org/10.1103/PhysRevD.96.072002. arXiv: 1703.09665

44. CMS Collaboration, Measurement of the inclusive jet cross section in $p p$ collisions at $\sqrt{s}=7 \mathrm{TeV}$. Phys. Rev. Lett. 107, 132001 (2011). https://doi.org/10.1103/PhysRevLett.107. 132001. arXiv: 1106.0208

45. CMS Collaboration, Measurement of the inclusive production cross sections for forward jets and for dijet events with one forward and one central jet in pp collisions at $\sqrt{s}=7 \mathrm{TeV}$. JHEP 06, 036 (2012). https://doi.org/10.1007/JHEP06(2012)036. arXiv: 1202.0704

46. CMS Collaboration, Measurements of differential jet cross sections in proton-proton collisions at $\sqrt{s}=7 \mathrm{TeV}$ with the CMS detector. Phys. Rev. D 87, 112002 (2013). https://doi.org/10.1103/ PhysRevD.87.112002. arXiv:1212.6660

47. CMS Collaboration, Measurement of the ratio of inclusive jet cross sections using the anti- $k_{T}$ algorithm with radius parameters $R=0.5$ and 0.7 in $p p$ collisions at $\sqrt{s}=7 \mathrm{TeV}$. Phys. Rev. D 90, 072006 (2014). https://doi.org/10.1103/PhysRevD.90. 072006. arXiv:1406.0324

48. CMS Collaboration, Measurement of the inclusive jet cross section in $p p$ collisions at $\sqrt{s}=2.76 \mathrm{TeV}$. Eur. Phys. J. C 76, 265 (2016). https://doi.org/10.1140/epjc/s10052-016-4083-z. arXiv: 1512.06212

49. CMS Collaboration, Measurement of the double-differential inclusive jet cross section in proton-proton collisions at $\sqrt{s}=13$
TeV. Eur. Phys. J. C 76(8), 451 (2016). https://doi.org/10.1140/ epjc/s10052-016-4286-3. arXiv:1605.04436

50. CMS Collaboration, Measurement and QCD analysis of doubledifferential inclusive jet cross-sections in pp collisions at $\sqrt{s}=8$ $\mathrm{TeV}$ and ratios to 2.76 and $7 \mathrm{TeV}$. JHEP03, 156 (2017). https:// doi.org/10.1007/JHEP03(2017)156. arXiv:1609.05331

51. S.D. Ellis, Z. Kunszt, D.E. Soper, One-jet inclusive cross section at order $\alpha_{s}^{3}$ : quarks and gluons. Phys. Rev. Lett. 64, 2121 (1990). https://doi.org/10.1103/PhysRevLett.64.2121

52. S.D. Ellis, Z. Kunszt, D.E. Soper, Two-jet production in hadron collisions at order $\alpha_{s}^{3}$ in QCD. Phys. Rev. Lett. 69, 1496 (1992). https://doi.org/10.1103/PhysRevLett.69.1496

53. W.T. Giele, E.W.N. Glover, D.A. Kosower, The two-jet differential cross section at $\mathcal{O}\left(\alpha_{s}^{3}\right)$ in hadron collisions. Phys. Rev. Lett. 73, 2019 (1994). https://doi.org/10.1103/PhysRevLett.73.2019. arXiv:hep-ph/9403347

54. M.H. Seymour, Jet shapes in hadron collisions: higher orders, resummation and hadronization. Nucl. Phys. B 513, 269 (1998). https://doi.org/10.1016/S0550-3213(97)00711-6. arXiv:hep-ph/9707338

55. G.C. Blazey et al., Run II jet physics. In: Proceedings, Physics at Run II: QCD and Weak Boson Physics Workshop, p. 47. Batavia, IL, USA, March 4-6, June 3-4, November 4-6, 1999. arXiv:hep-ex/0005012

56. G.P. Salam, Towards jetography. Eur. Phys. J. C 67, 637 (2010). https://doi.org/10.1140/epjc/s10052-010-1314-6. arXiv:0906.1833

57. ZEUS Collaboration, An NLO QCD analysis of inclusive crosssection and jet-production data from the ZEUS experiment. Eur. Phys. J. C 42, 1 (2005). https://doi.org/10.1140/epjc/ s2005-02293-x. arXiv:hep-ph/0503274

58. H1 and ZEUS Collaboration, Combination of measurements of inclusive deep inelastic $e^{ \pm} p$ scattering cross sections and QCD analysis of HERA data. Eur. Phys. J. C 75, 580 (2015). https:// doi.org/10.1140/epjc/s10052-015-3710-4. arXiv:1506.06042

59. R.D. Ball, Precision NNLO determination of $\alpha_{S}\left(M_{Z}\right)$ using an unbiased global parton set. Phys. Lett. B 707, 66 (2012). https:// doi.org/10.1016/j.physletb.2011.11.053. arXiv:1110.2483

60. L.A. Harland-Lang, A.D. Martin, P. Motylinski, R.S. Thorne, Parton distributions in the LHC era: MMHT 2014 PDFs. Eur. Phys. J. C 75, 204 (2015). https://doi.org/10.1140/epjc/ s10052-015-3397-6. arXiv:1412.3989

61. D0 Collaboration, Determination of the strong coupling constant from the inclusive jet cross section in $p \bar{p}$ collisions at $\sqrt{s}=1.96$ TeV. Phys. Rev. D 80, 111107 (2009). https://doi.org/10.1103/ PhysRevD.80.111107. arXiv:0911.2710

62. CMS Collaboration, Constraints on parton distribution functions and extraction of the strong coupling constant from the inclusive jet cross section in pp collisions at $\sqrt{s}=7 \mathrm{TeV}$. Eur. Phys. J. C 75, 288 (2015). https://doi.org/10.1140/epjc/ s10052-015-3499-1. arXiv:1410.6765

63. CDF Collaboration, Measurement of the strong coupling constant from inclusive jet production at the Tevatron $\bar{p} p$ collider. Phys. Rev. Lett. 88, 042001 (2002). https://doi.org/10.1103/ PhysRevLett.88.042001. arXiv:hep-ex/0108034

64. W.T. Giele, E.W.N. Glover, J. Yu, The determination of $\alpha_{S}$ at hadron colliders. Phys. Rev. D 53, 120 (1996). https://doi.org/10. 1103/PhysRevD.53.120. arXiv:hep-ph/9506442

65. CDF Collaboration, Inclusive jet cross-section in $\bar{p} p$ collisions at $\sqrt{s}=1.8$ TeV. Phys. Rev. Lett. 68, 1104 (1992). https://doi.org/ 10.1103/PhysRevLett.68.1104

66. B. Malaescu, P. Starovoitov, Evaluation of the strong coupling constant $\alpha_{S}$ using the ATLAS inclusive jet cross-section data. Eur. Phys. J. C 72, 2041 (2012). https://doi.org/10.1140/epjc/ s10052-012-2041-y. arXiv:1203.5416 
67. T. Biekötter, M. Klasen, G. Kramer, Next-to-next-to-leading order contributions to inclusive jet production in deep-inelastic scattering and determination of $\alpha_{s}$. Phys. Rev. D 92(7), 074037 (2015). https://doi.org/10.1103/PhysRevD.92.074037. arXiv: 1508.07153

68. S.D. Ellis, D.E. Soper, Successive combination jet algorithm for hadron collisions. Phys. Rev. D 48, 3160 (1993). https://doi.org/ 10.1103/PhysRevD.48.3160. arXiv:hep-ph/9305266

69. M. Cacciari, G.P. Salam, G. Soyez, The anti- $k_{t}$ jet clustering algorithm. JHEP 04, 063 (2008). https://doi.org/10.1088/1126-6708/ 2008/04/063. arXiv:0802.1189

70. R.K. Ellis, W.J. Stirling, B.R. Webber, QCD and Collider Physics. Nuclear Physics and Cosmology, Cambridge Monographs on Particle Physics (Cambridge University Press, Cambridge, 1996)

71. S. Dittmaier, A. Huss, C. Speckner, Weak radiative corrections to dijet production at hadron colliders. JHEP 11, 095 (2012). https:// doi.org/10.1007/JHEP11(2012)095. arXiv:1210.0438

72. R. Frederix et al., The complete NLO corrections to dijet hadroproduction. JHEP 04, 076 (2017). https://doi.org/10.1007/ JHEP04(2017)076. arXiv:1612.06548

73. A. Buckley, General-purpose event generators for LHC physics. Phys. Rep. 504, 145 (2011). https://doi.org/10.1016/j.physrep. 2011.03.005. arXiv: 1101.2599

74. Z. Nagy, Three jet cross-sections in hadron hadron collisions at next-to-leading order. Phys. Rev. Lett. 88, 122003 (2002). $\quad$ https://doi.org/10.1103/PhysRevLett.88.122003. arXiv:hep-ph/0110315

75. Z. Nagy, Next-to-leading order calculation of three-jet observables in hadron hadron collisions. Phys. Rev. D 68, 094002 (2003). https://doi.org/10.1103/PhysRevD.68.094002. arXiv:hep-ph/0307268

76. T. Kluge, K. Rabbertz, M. Wobisch, fastNLO: Fast pQCD calculations for PDF fits, in 14th International Workshop on Deep Inelastic Scattering (DIS 2006), p. 483. Tsukuba, Japan, April 20-24, 2006. arXiv:hep-ph/0609285

77. D. Britzger, K. Rabbertz, F. Stober, M. Wobisch, New features in version 2 of the fastNLO project, in Proceedings, XX. International Workshop on Deep-Inelastic Scattering and Related Subjects (DIS 2012), p. 217. Bonn, Germany, March 26-30, (2012). arXiv:1208.3641. https://doi.org/10.3204/ DESY-PROC-2012-02/165

78. M. Cacciari, G.P. Salam, G. Soyez, FastJet user manual. Eur. Phys. J. C 72, 1896 (2012). https://doi.org/10.1140/epjc/ s10052-012-1896-2. arXiv:1111.6097

79. M.R. Whalley, D. Bourilkov, R.C. Group, The Les Houches Accord PDFs (LHAPDF) and LHAGLUE, in Proceedings, HERA and the LHC: A Workshop on the Implications of HERA for LHC Physics: Vol. B, Geneva, Switzerland and Hamburg, Germany, March 26-27, October 11-13, March 21-24, 2004-2005. arXiv:hep-ph/0508110

80. A. Buckley et al., LHAPDF6: parton density access in the LHC precision era. Eur. Phys. J. C 75, 132 (2015). https://doi.org/10. 1140/epjc/s10052-015-3318-8. arXiv:1412.7420

81. K.G. Chetyrkin, J.H. Kuhn, M. Steinhauser, RunDec: a mathematica package for running and decoupling of the strong coupling and quark masses. Comput. Phys. Commun. 133, 43 (2000). https://doi.org/10.1016/S0010-4655(00)00155-7. arXiv:hep-ph/0004189

82. B. Schmidt, M. Steinhauser, CRunDec: a C++ package for running and decoupling of the strong coupling and quark masses. Comput. Phys. Commun. 183, 1845 (2012). https://doi.org/10.1016/j.cpc. 2012.03.023. arXiv: 1201.6149

83. G't Hooft, M .J .G. Veltman, Regularization and renormalization of gauge fields. Nucl. Phys. B 44, 189 (1972). https://doi.org/10. 1016/0550-3213(72)90279-9
84. G' $\mathrm{t}$ Hooft, Dimensional regularization and the renormalization group. Nucl. Phys. B 61, 455 (1973). https://doi.org/10.1016/ 0550-3213(73)90376-3

85. W.A. Bardeen, A.J. Buras, D.W. Duke, T. Muta, Deep Inelastic scattering beyond the leading order in asymptotically free gauge theories. Phys. Rev. D 18, 3998 (1978). https://doi.org/10.1103/ PhysRevD.18.3998

86. J. Currie et al., Single jet inclusive production for the individual jet $p_{T}$ scale choice at the LHC. Acta Phys. Polon. B 48, 955 (2017). https://doi.org/10.5506/APhysPolB.48.955. arXiv:1704.00923 (23rd Cracow Epiphany Conference on Particle Theory Meets the First Data from LHC Run 2, Krakow, Poland, 9-12 January 2017)

87. J. Currie, Infrared sensitivity of single jet inclusive production at hadron colliders. JHEP 10, 155 (2018). https://doi.org/10.1007/ JHEP10(2018)155. arXiv:1807.03692

88. M. Miller, Private communication (2006)

89. H.-L. Lai et al., New parton distributions for collider physics. Phys. Rev. D 82, 074024 (2010). https://doi.org/10.1103/ PhysRevD.82.074024. arXiv:1007.2241

90. A.D. Martin, W.J. Stirling, R.S. Thorne, G. Watt, Parton distributions for the LHC. Eur. Phys. J. C 63, 189 (2009). https://doi.org/ 10.1140/epjc/s10052-009-1072-5. arXiv:0901.0002

91. NNPDF Collaboration, Parton distributions from high-precision collider data. Eur. Phys. J. C 77(10), 663 (2017). https://doi.org/ 10.1140/epjc/s10052-017-5199-5. arXiv:1706.00428

92. L.A. Harland-Lang, A.D. Martin, R.S. Thorne, The impact of LHC jet data on the MMHT PDF fit at NNLO. Eur. Phys. J. C 78(3), 248 (2018). https://doi.org/10.1140/epjc/ s10052-018-5710-7. arXiv:1711.05757

93. S. Dulat et al., New parton distribution functions from a global analysis of quantum chromodynamics. Phys. Rev. D 93, 033006 (2016). https://doi.org/10.1103/PhysRevD.93.033006. arXiv: 1506.07443

94. CDF and D0 Collaboration, Combination of CDF and D0 results on the mass of the top quark using up to $9.7 / \mathrm{fb}^{-1}$ at the Tevatron. arXiv: 1407.2682

95. ATLAS and CMS Collaboration, Jet energy scale uncertainty correlations between ATLAS and CMS at $8 \mathrm{TeV}$. Technical report ATL-PHYS-PUB-2015-049, CMS-PAS-JME-15-001, CERN (2015)

96. ATLAS and CMS Collaboration, Jet energy scale uncertainty correlations between ATLAS and CMS. Technical report ATLPHYS-PUB-2014-020, CMS-PAS-JME-14-003, CERN (2014)

97. S. Alekhin, J. Blümlein, S. Moch, R. Placakyte, Parton distribution functions, $\alpha_{s}$ and heavy-quark masses for LHC Run II. Phys. Rev. D 96(1), 014011 (2017). https://doi.org/10.1103/PhysRevD.96. 014011. arXiv: 1701.05838

98. S. Alekhin, J. Blümlein, S. Moch, NLO PDFs from the ABMP16 fit. Eur. Phys. J. C 78(6), 477 (2018). https://doi.org/10.1140/epjc/ s10052-018-5947-194. arXiv: 1803.07537

99. NNPDF Collaboration, Parton distributions for the LHC Run II. JHEP 04, 040 (2015). https://doi.org/10.1007/JHEP04(2015)040. arXiv: 1410.8849

100. F. James, M. Roos, Minuit: a system for function minimization and analysis of the parameter errors and correlations. Comput. Phys. Commun. 10, 343 (1975). https://doi.org/10.1016/ 0010-4655(75)90039-9

101. J. Butterworth et al., PDF4LHC recommendations for LHC Run II. J. Phys. G 43, 023001 (2016). https://doi.org/10.1088/ 0954-3899/43/2/023001. arXiv: 1510.03865

102. S. Catani, D. de Florian, M. Grazzini, P. Nason, Soft gluon resummation for Higgs boson production at hadron colliders. JHEP 07, 028 (2003). https://doi.org/10.1088/1126-6708/2003/07/028. arXiv:hep-ph/0306211 
103. M. Cacciari, The $t$ anti- $t$ cross-section at $1.8 \mathrm{TeV}$ and $1.96 \mathrm{TeV}$ : a study of the systematics due to parton densities and scale dependence. JHEP 04, 068 (2004). https://doi.org/10.1088/1126-6708/ 2004/04/068. arXiv:hep-ph/0303085
104. A. Banfi, G.P. Salam, G. Zanderighi, Phenomenology of event shapes at hadron colliders. JHEP 06, 038 (2010). https://doi.org/ 10.1007/JHEP06(2010)038. arXiv:1001.4082 